\title{
A DYNAMIC THEORY OF REGULATORY CAPTURE
}

\author{
Alessandro De Chiara
}

Marco A. Schwarz 
Title: A dynamic theory of regulatory capture

Abstract: Firms often try to influence individuals that, like regulators, are tasked with advising or deciding on behalf of a third party. In a dynamic regulatory setting, we show that a firm may prefer to capture regulators through the promise of a lucrative future job opportunity (i.e., the revolving-door channel) than through a hidden payment (i.e., a bribe). This is because the revolving door publicly signals the firm's eagerness and commitment to rewarding lenient regulators, which facilitates collusive equilibria. We find that opening the revolving door conditional on the regulator's report is usually more efficient than a blanket ban on post-agency employment and may increase social welfare. This insight extends to a variety of applications and can also be used to determine the optimal length of cooling-off periods.

JEL Codes: D73, D86, H11, J45, L51

Keywords: Corruption, dynamic games, regulatory capture, revolving door

\section{Authors:}

Alessandro De Chiara

Marco A. Schwarz

Universitat de Barcelona, BEAT University of Innsbruck, CESifo

Email: aledechiara@ub.edu_ Email: marco.schwarz@uibk.ac.at

Date: March 2021

Acknowledgements: We wish to thank Øyvind Aas, Benito Arruñada, Jean-Etienne de Bettignies, Jacopo Bizzotto, Alessandra Fenizia, Rosa Ferrer, Marc Kaufmann, Botond Kőszegi, Ester Manna, Robert Somogyi, and Adam Szeidl as well as audiences at the Universitat de les Illes Balears (Palma de Mallorca, Spain), Budapest University of Technology and Economics, Oslo Metropolitan University (Norway), Central European University (Budapest, Hungary), Online Oligoworkshop 2020, 6th Workshop on Relational Contracts (Moscow, Russia, online), and Erasmus University Rotterdam (online) for helpful comments. Financial support from the Austrian Science Fund (SFB F63) is gratefully acknowledged. 


\section{Introduction}

Firms often have an incentive to influence individuals tasked with deciding on behalf of or advising a third party. Most prominently, firms may benefit from swaying the behavior of their regulators, which can distort economic outcomes (e.g., see Stigler, 1971 and Laffont and Tirole, 1991, 1993). To do so, firms can promise lucrative future job opportunities to lenient regulatory officials. This practice, known as revolving door, appears to be rife in developed countries and has long been the subject of a lively debate over the opportunity of adopting measures to limit the transition of regulators to the private sector. Alternatively, firms could directly pay a bribe to regulators in exchange for their leniency. These different channels are often viewed as substitute tools for firms to capture their regulators.

In this paper, we develop a dynamic model to shed light on a crucial distinction between bribes and the revolving door as means to influence the regulatory outcome, which is tied to their different informational content and that has been overlooked so far. The intuition is the following. Because formal exchanges of favors between regulatory officials and regulated firms are prohibited, rewarding a friendly official can only take the form of an informal promise. As there is no guarantee that the firm will keep its end of the bargain, the regulator may refuse to be swayed. A firm may tremendously benefit from publicly rewarding a compliant regulator, as this could signal to future regulators that being accommodating pays off. Since the firm need not conceal its hiring decisions, the revolving door can convey information about the firm's eagerness and commitment to reward friendly officials. Therefore, the public nature of the recruitment decision helps facilitate collusive equilibria. By contrast, bribes are inherently private in nature because of their illegality and, as such, do not help coordinate the firm's and the regulators' behavior. Making the bribe publicly observable is also unappealing to the firm since it may trigger an investigation and, ultimately, overturn a favorable regulatory decision. Conversely, the firm could convincingly argue in court that the reason behind hiring a former regulator is his skillfulness and knowledge rather than his past lenient monitoring.

There is an additional, related reason for why bribes may be a poor substitute for the revolving door: bribes necessarily require some explicit agreement between the collusive partners. This implies that general anti-corruption practices, such as a whistleblowing protection policy or a close scrutiny of the firm-regulator interaction, can successfully deter 
bribery. By contrast, the revolving-door channel may as well work implicitly: observing past behavior and following industry norms suffice to inform the parties of the gains that can be obtained by colluding. The absence of smoking-gun evidence renders standard anti-corruption policies ineffective.

Although closing the revolving door can solve the problem of regulatory capture, we caution that it can give rise to other inefficiencies, as already pointed out in the literature and by many observers (e.g., Che, 1995). Firstly, it might actually be socially efficient that some regulatory officials join the regulated firms as they could bring their industry knowhow and expertise to the private sector. Secondly, regulators may demand a lower salary in the anticipation of landing highly-paid positions in the private sector after having gained regulatory experience. These benefits would be lost if the revolving door were closed. We demonstrate that a conditional restriction on post-agency employment can mitigate these inefficiencies as it ensures that skilled regulators do not necessarily lose out on appealing career opportunities, while at the same time it preserves the benefit of deterring regulatory capture.

In the model, introduced in Section 2, we consider an infinitely repeated game between a firm and a benevolent principal, where the latter can be thought of as the policymaker. In every period, a regulatory decision that affects the firm's payoff and social welfare must be taken. The principal designs the regulatory policy, but must delegate the collection of information about a time-varying state variable, which is critical for the regulatory decision, to a regulatory agency. To carry out its monitoring activities, the regulatory agency employs experts who, unlike the firm and the principal, are short-lived. The experts may sometimes be able to hide and not report the collected evidence. The principal can offer experts a bonus contract that rewards them for reporting evidence and/or close the revolving door, that is, prohibiting them from joining the regulated firm afterward.

In Section 3, we show that the revolving door can be kept open if the firm-agency interaction is one-off or if it is repeated but the firm is not patient enough. If so, the firm's promise of rewarding an accommodating expert is not credible. Conversely, when interaction is repeated and the firm is sufficiently patient, the promise of a future employment may influence the experts' reports. The principal can deter regulatory capture by offering a high bonus for reporting evidence or by closing the revolving door. In the 
latter case, we highlight that the revolving door should only be closed if the expert fails to provide evidence: such policy, that we refer to as conditional ban, effectively deters regulatory capture while retaining part of the benefits associated with the transfer of regulatory expertise from the agency to the private sector. Notably, the conditional ban is especially valuable when the firm is very patient or when there is frequent firm-agency interaction: in that case, the firm would be willing to offer a high salary to accommodating experts, thereby making it overly expensive for the principal to use a bonus scheme to deter capture.

Our basic mechanism and chief policy prescription extend to a variety of settings, including environments in which the firm can observe or affect the state of the world (see Subsection 4.1 and Subsection 4.3, respectively), or where an expert must exert effort to collect any information (Section 4.2). The conditional ban proves particularly appealing when the expert must incur an effort cost to collect evidence, whereas it is less appealing when the firm can affect the state of the world with its actions. In the latter case, a conditional ban provides perverse incentives for the firm to under-invest so that it can hire an expert more often.

Moreover, we analyze a number of extensions that let us unveil additional results that may be helpful in designing policies. Firstly, we highlight in Subsection 4.4 that the principal may prefer to hire experts who are less able to obtain an accurate signal about the state of the world so as to reduce the firm's temptation to engage in capture or lower the cost of capture deterrence.

Secondly, rather than prohibiting an expert from ever joining the private sector, the principal may opt for a temporary restriction, i.e., a cooling-off period. In our setting, we find that a finite cooling-off period will be used only to reduce the cost of the bonus scheme and, if adopted, its length should be conditional on the expert's report. Capture becomes less appealing to the firm-expert coalition when sending a report favorable to the firm kicks in the employment restriction. For this reason, a conditional cooling-off period reduces the bonus needed to induce truthful reporting. Moreover, we show that wrongly timed cooling-off periods may hurt all involved players and we caution that cooling-off periods uniform across many regulatory agencies and, hence, industries are unlikely to be optimal (see Subsection 4.5). 
Thirdly, although the baseline model assumes that an expert only makes one report, in Subsection 4.6 we show that the gist of our results carries over to an environment wherein each expert interacts several times with the regulated firm when working for the agency and sends reports sequentially. We show that it may suffice to condition the ban on the content of some and not all the reports. When this is the case, the ban should better be made contingent on the content of early rather than late reports. To understand why, consider that the firm may always try to capture an expert for the first reports if only late reports are considered to determine whether the revolving door is open or not. Conversely, if early reports are critical to determining an expert's chance of joining the regulated firm afterward, the firm can try to engage in capture only when the first reports were unfavorable.

Lastly, in Subsection 4.7, we allow for an exogenous enforcement mechanism that compels the experts and the firm to abide by what they agree upon in an explicit sidecontract. We find that the firm will resort to either bribes or the revolving door to capture experts and that the former will be used whenever their associated transaction cost is sufficiently low, even when players are very patient so that the firm could also use the revolving-door channel. Our analysis highlights the importance of complementarities among anti-capture policies: some measures that deter bribery (respectively, the use of the revolving door) may in fact lead the firm to use the revolving door (use bribes) to capture experts. Our results may account for why bribery is prevalent in the developing world, whereas the revolving door is rife in wealthier countries. In the former, experts' conduct is often ineffectively monitored and there is limited social stigma associated with explicit corruption. In the latter, superior monitoring, better enforcement, and social norms mitigate the threat of bribery, but the longer life-span of firms reinforces the credibility of promises to reward lenient experts.

Section 5 provides some concluding remarks. All proofs are relegated to the appendix. We now discuss the related theoretical literature and empirical evidence.

Related theoretical literature. The economics literature has long recognized how the risk of collusion between a firm and the regulator entrusted with its supervision can undermine the regulatory outcome. The contract-theory literature pioneered by Tirole (1986) shows that collusion can be prevented by rewarding the regulatory officials for 
revealing information which can hurt the firm. ${ }^{1}$ In this strand of the literature, it is typically assumed that the firm and the agency can strike an enforceable collusive agreement. ${ }^{2}$ One of the purposes of our model is to focus on one of the channels that can make such collusive agreement self-enforceable, namely reputation, and study in great detail its repercussions for regulation outcomes and for the design of policies to prevent capture or alleviate its most harmful effects. In this regard, our paper is linked to Martimort (1999), who also analyzes the dynamics of regulation. Like him, we consider self-enforcing agreements between the firm and the regulator and we posit that the principal cannot commit to future transfers to the regulator. There are some relevant modeling differences as Martimort (1999) assumes that all players are long-lived and characterizes the collusive-proof regulatory contract, whereas in our model the principal may prefer not to prevent capture and there is an infinite stream of short-lived regulators. More fundamentally, the goals of the papers are different. Martimort (1999) aims to explain why the regulatory process becomes less efficient over time and to endogenize the transaction costs of side-contracts. By contrast, we are interested in studying the mechanism whereby regulators are swayed by the firms and we highlight the trade-offs that preventing capture bring about.

Some theoretical papers have explicitly focused on both the upsides and the drawbacks of the revolving-door practice. Salant (1995) shows how revolving doors could facilitate cooperation between managers and regulators, leading to higher investments and increasing social welfare. Che (1995) studies the relationship between a regulator's performance and revolving doors. He shows how post-agency employment opportunities can affect the regulator's ex-ante incentives to acquire regulatory expertise and his ex-post incentives to favor the regulated firm. We also highlight the pros and cons of revolving door and we also come to the conclusion that tolerating collusion may be beneficial, though for different reasons. Specifically, allowing collusion may prompt monitoring effort in Che (1995), whereas it can reduce the regulatory cost in our paper. However, Che (1995) considers a one-off interaction between the players and the revolving door is only one of the possible

\footnotetext{
${ }^{1}$ Recent contributions to the design of regulation in the presence of corruption concerns include Drugov (2010), Hiriart et al. (2010), Hiriart and Martimort (2012), Angelucci and Russo (2017), Ortner and Chassang (2018), and De Chiara and Manna (2019).

${ }^{2}$ Mechanisms such as emotions, reciprocity, and reputation are often invoked to justify the enforceability of this side-contract (for instance, see Tirole, 1992).
} 
mechanisms that the firm and the regulator can rely on to collude in his model, whereas it plays a more prominent role in our paper, as formally shown by distinguishing between different channels.

Although the foci of the papers are different, the basic mechanism in our model shares some similarities with the one in Chassang and Padró I Miquel (2019). They show that publicly available information enables a firm to credibly threaten to retaliate against a whistleblower, whereas we highlight how a firm could use the principal's authorization decision to credibly and publicly reward a lenient expert. Moreover, like intimidation in their model, in ours capture does not require an explicit side-agreement between the firm and the expert.

There is also a link with two recent papers that develop dynamic games. First, TroyaMartinez and Wren-Lewis (2018) study relational contracts between an agent and a supervisor where there is room for corruption, highlighting the differences with more standard principal-agent models. Second, Barron and Guo (2021) study the related issue of extortion in a model where a principal interacts with an infinite stream of agents. The agents' ability to commit to public messages enables agents to blackmail the principal and can fully destroy cooperation.

Moreover, this paper contributes to the credence goods literature (Darby and Karni, 1973; Dulleck and Kerschbamer, 2006). In particular, it extends the small literature that views the policymaker as the party that is seeking advice from an expert (Dulleck et al., 2015). It also adds to the literature on commissions and kickbacks (Inderst and Ottaviani, 2012a,b,c, and Inderst, 2015) in the sense that another party - here the firm may influence the expert's choice.

Empirical evidence. There is a growing body of empirical evidence investigating the effects of revolving door, thanks to the increasing availability of detailed datasets on workers' transition from regulatory agencies to the private sector (and vice versa). There is evidence that the revolving door is both associated with capture and incentives to acquire or signal expertise and skills that can result in superior regulatory outcomes. Concerning suggestive evidence of capture, Tabakovic and Wollmann (2018) study the career trajectory of examiners working for the USPTO who evaluate the patent applications filed by law firms on behalf of inventors. Among other things, they find that (i) examin- 
ers award more patents to the law firms they end up working for; (ii) the difference is significantly less pronounced when the likelihood of being hired is lower for exogenous reasons (e.g., recessions); (iii) the patents awarded to the law firms they later work for receive substantially fewer citations, indicating that these applications are held to a lower standard. Recently, suggestive evidence of regulatory capture has been found in the insurance solvency regulation (Tenekedjieva, 2019), for credit rating analysts (Cornaggia et al., 2016), and even for scientists working for the FDA advisory committees and panels (Piller, 2018). Notably, firms-regulators interaction is regular and relatively frequent in all these industries: over the 15 years considered by Tabakovic and Wollmann (2018), at least six law firms filed more than 10,000 applications (and at least 21 law firms filed more than 1,000); FDA advisory committees regularly meet with representatives of large pharmaceutical corporations, and credit rating analysts rate securities of large underwriting merchant banks. Lastly, while financial exams of insurance firms may be ordered less frequently, they must be performed at least once every five years.

Other papers have found evidence pointing to an incentive effect: for instance, Kempf (2020) finds that more accurate credit rating analysts working for Moody's are more likely to join the underwriters. ${ }^{3}$ Focusing on Brazilian health procurement, Barbosa and Straub (2017) find that civil servants who later join private providers purchase products at a lower price, whereas the transition of workers from providers to the administration appears detrimental as it is associated with larger purchases from the connected provider at higher prices. DeHaan et al. (2015) find that more aggressive enforcement effort (e.g., collecting higher damages or seeking criminal proceedings against companies accused of accounting misrepresentation) are weakly associated with those SEC lawyers who later join law firms, especially those who specialize in SEC enforcement cases, although those lawyers who will be based in Washington, D.C., tend to be laxer.

Also relevant for our paper is the empirical literature on the lobbying process which has shown that, besides their technical knowledge and expertise, lobbyists are valuable in that they bring connections, namely special interest access to legislators (Bertrand et al., 2014). Accordingly, prior experience in the federal government is especially sought-after

\footnotetext{
${ }^{3}$ Kempf (2020) also finds some evidence of capture as more optimistic analysts on some specific deals, that is, those who give ratings that are biased upwardly as compared to their counterparts at S\&P and Fitch, are more likely to be hired by one of the underwriting investment banks.
} 
because of the network of friends and colleagues that a revolving-door lobbyist may have developed (Blanes i Vidal et al., 2012). We put forward a complementary mechanism that renders the recruitment of former regulators or government employees particularly appealing: it enables the regulated firm to signal its intent to reward accommodating regulators (the creation of the industry norm) and ensures the monitoring of its promises, which is critical for sustaining a collusive equilibrium.

\section{Model}

We consider a model with an infinitely-lived, profit-maximizing firm (it), an infinitelylived, welfare-maximizing principal (she), and an infinite stream of wealth-constrained, short-lived experts (he). In each period, the firm can produce a new good that generates a private benefit $G>0$. The principal decides whether or not to authorize production, since this can cause unverifiable damages to third parties. Specifically, if the state is safe, i.e., $\theta=S$, damages do not occur. If the state is unsafe, i.e., $\theta=U$, production generates damages $D>0$. The state is unsafe with probability $q>0$ and we assume that:

$$
D>G>q D .
$$

Therefore, it would be socially desirable if production were authorized only in the safe state and, without knowing the state, it would be better to authorize production. Whether production brings about damages is not known, whereas the probability distribution of the states of the world is common knowledge.

The principal regulates the firm through a dedicated agency. In every period, an expert is employed at the agency and tasked with collecting a signal about $\theta$. If $\theta=S$, the signal is always uninformative, i.e., $s=\emptyset$. Conversely, if $\theta=U$, the signal reveals the true state of the world. The signal is privately observed by the expert, whereas the principal and the firm only know its distribution. The expert sends a public report $r$ to the principal. We assume that the expert cannot forge information, that is, if $s=\emptyset, r=\emptyset$, which is the case if the expert needs to provide verifiable evidence along with a report $r$. However, if $s=U$, the expert can conceal information at a private concealment cost $c$, whose magnitude depends on the nature of the information and thus becomes known only after the expert has collected the signal. For simplicity, we assume that this cost 
can take two levels, $c \in\{\underline{c}, \infty\}$, with $\underline{c} \geq 0$. It is common knowledge that $c=\infty$ with probability $\eta \in(0,1)$. We say that (regulatory) capture occurs when the expert reports $r=\emptyset$, although $s=U$.

At the beginning of the game, the principal commits to an authorization policy as a function of the report, and this decision is denoted $x_{r} \in\{0,1\}$, where $x=1$ if production is allowed and $x=0$ if production is prohibited. The principal also pays a bonus $\beta_{r} \geq 0$ to the experts, which is contingent on the report. ${ }^{4}$ This payment generates a socially costly shadow cost of public funds $\lambda \beta_{r}$, where $\lambda \geq 0$, that can be due to inefficiencies in tax collection or to the political resentment over rewards paid to civil servants. ${ }^{5} \mathrm{We}$ assume that the principal cannot commit to a sequence of regulatory transfers.

After sending the report, the expert can seek post-agency employment. The firm can make a wage offer $w$, possibly as a function of the report that the expert made. We assume that the expert makes a positive contribution to the industry after having gathered experience in the regulatory agency. Specifically, an expert increases the firm's profits by $v$ and could yield an outcome $\bar{u}$ to other public or private institutions in the industry, with $v \geq \bar{u} \geq 0$. Plausibly, while both the regulated firm and other organizations in the same industry would be eager to employ an expert who has extensive knowledge of the regulatory procedures, the former may especially value the expert's relative familiarity with the firm's production process. Thanks to competition for the expert's services, his outside option after leaving the agency is equal to $\bar{u}$. We will refer to $v$ as the value of the regulatory expertise.

The timing of the stage game is as follows:

0. The principal commits to the authorization decision as a function of the report $x_{r} \in\{0,1\}$.

1. Nature draws the state of the world $\theta_{t}$ and the concealment cost $c_{t}$. The principal sets the bonus scheme for the expert, $\beta_{t}\left(r_{t}\right) \geq 0$.

2. If the expert accepts to work for the regulatory agency, he observes the signal $s_{t}$ and sends a public report $r_{t}$ to the principal who authorizes or not production according

\footnotetext{
${ }^{4}$ This reward can also come in the form of a promotion or a permanent salary increase.

${ }^{5}$ In many applications, the principal cannot make performance-dependent bonus payments (e.g., to civil servants). Our model (with $\lambda$ very large) encompasses these cases.
} 
to $x_{r}$ and pays the expert $\beta_{t}\left(r_{t}\right)$.

3. The firm may make an offer $w_{t}$ to the expert, who can either accept this offer or take up his outside option.

The repeated game we analyze involves the infinite repetition of the stage game starting at stage 1 . The principal and the firm use the same discount factor $\delta<1$, and we multiply each period's payoff by $1-\delta$ so as to obtain per-period averages. We will occasionally interpret a small $\delta$ as infrequent interactions between the firm and the regulatory agency. ${ }^{6}$

We conclude this section by commenting on some of the modelling assumptions. Firstly, the assumption that the signal $s$ perfectly reveals the state of the world is solely made to save on notation: qualitatively, all the main results carry trough if an expert observes a signal that is not fully informative. Secondly, we assume that experts are valuable to the regulated firm as well as other organizations in the industry after having worked for the agency. Similarly, in the model of Bond and Glode (2014), working in a regulatory agency leads to the accumulation of human capital that firms value. There is also some empirical evidence supporting the human-capital accumulation formation hypothesis (Blanes i Vidal et al., 2012). Lastly, akin to Martimort (1999), and for the same purpose, we assume that the principal has limited commitment ability: she can commit to an authorization policy but not to the wage policy. It stands to reason that the latter cannot be modified as easily as the former. Its implication is that the principal cannot preempt capture by committing to paying a large reward to a future expert to induce his deviation from a collusive equilibrium. Such a reward would not be credible and, as a result, if the principal wants to use the bonus scheme to prevent capture in period $t$, she must offer a reward to that period's expert.

\section{Regulatory Capture via the Revolving Door}

In this section, we impose that the principal cannot prevent the expert from joining the firm after leaving the agency. We relax this assumption in Section 3.1.

\footnotetext{
${ }^{6}$ This is admittedly a loose interpretation of the discount factor. What we have in mind is that if the players interact every $n$ periods, the firm and the principal will discount the next interaction by $\delta^{n}$, which is the lower the higher $n$, and thus, the more infrequent those interactions are.
} 
First, note that the principal could obtain social welfare $W^{N O}=G-q D$ if she did not hire any expert. Regulation is thus valuable in that it may bring about a benefit equal to $q(D-G)+v$, which is attained if the principal sets $x_{U}=0<1=x_{\emptyset}$, the experts always report truthfully the observed signal, and they later join the regulated firm. If the firm's interaction with the regulatory agency is one-off, $W^{F B}=(1-q) G+v$, where $F B$ stands for first-best, is indeed the level of social welfare that regulation achieves without having to reward an expert to induce truthful revelation. To see this, solve the stage game backwards. In stage 3, the firm would always recruit the expert by offering a salary that matches the one that other organizations in the industry would pay, i.e., $w=\bar{u}$. In stage 2, every expert would choose the report that maximizes his utility, which depends on the bonus scheme and the concealment cost. To induce truthful revelation of the signal, the principal can offer $\beta_{\emptyset}=\beta_{U}=0$ in stage 1 and can announce $x_{\emptyset}=1$ and $x_{U}=0$ in stage 0 . The firm's expected profit in the stage-game is $\pi^{S G}=(1-q) G+v-\bar{u}$.

If the interaction with the regulatory agency is infinitely repeated, the firm may try to capture the experts so as to improve upon $\pi^{S G}$. Consider the following revolving-door implicit contract, denoted by $R D$, starting at date $t$ in which (i) experts always conceal unfavorable evidence when the concealment cost is low, $c=\underline{c}$, ; (ii) the firm rewards experts who report $r=\emptyset$ with improved post-agency job prospects; (iii) if at period $t+l$ the expert reported $r=\emptyset$ and the firm did not improve his job prospects, then at all $t+l^{\prime}$ with $l^{\prime} \geq l$, the experts always reveal unfavorable evidence. The revolving-door implicit contract is self-enforcing if these strategies form a Perfect Bayesian Equilibrium of the continuation game. ${ }^{7}$

For the revolving-door implicit contract to be an equilibrium, the post-agency salary must satisfy a set of participation and incentive constraints. In what follows, we restrict attention to stationary equilibria, ${ }^{8}$ and, as a result, we drop the period subscript $t$. A first necessary condition for the revolving-door implicit contract to be self-enforcing is that the following firm's dynamic capture-incentive compatibility constraint is satisfied:

$$
(1-\delta)\left(v-w_{\emptyset}\right)+\delta \pi^{R D} \geq(1-\delta)(v-\bar{u})+\delta \pi^{S G}
$$

\footnotetext{
${ }^{7}$ The notion of Perfect Bayesian Equilibrium enables us to consider experts' beliefs about the salaries they expect to receive from the firm. In equilibrium, experts will correctly anticipate the salary the firm will offer for any bonus schedule provided by the principal and for any report they make.

${ }^{8}$ In Appendix A, we show that this is without loss of generality.
} 
where $\pi^{R D}$ and $\pi^{S G}$ are the expected values of the streams of payoffs the firm obtains if regulatory capture does and does not take place, respectively. In the latter case, the firm receives the stage-game payoff in every period. If regulatory capture occurs, the firm obtains $G$ also whenever there is evidence that production is unsafe but the signal can be manipulated, that is, with probability $q(1-\eta)$. However, the firm must pay the salary $w_{\emptyset}$ whenever the report is favorable. As a result, the firm's expected profit by adhering to the revolving-door implicit contract is:

$$
\pi^{R D}=(1-q \eta)\left(G-w_{\emptyset}\right)-q \eta w_{U}+v
$$

It follows that:

$$
\Delta_{\pi} \equiv \pi^{R D}-\pi^{S G}=q(1-\eta) G-(1-q \eta) w_{\emptyset}-q \eta w_{U}+\bar{u} .
$$

Importantly, if the firm deviates, it still finds it profitable to hire the expert as his contribution to the firm's profit is positive. However, in that case, the firm will not pay him more than the expert's ex-post outside option $\bar{u}$.

A second necessary condition for the revolving-door implicit contract to describe an equilibrium is that the expert with a low concealment cost is willing to misreport evidence. We thus write the following expert's capture-incentive compatibility constraint:

$$
w_{\emptyset}-\underline{c}+\beta_{\emptyset} \geq w_{U}+\beta_{U}
$$

The firm will hire an expert that has reported $r=U$ with a salary $w_{U}=\bar{u}$ : in addition to being beneficial to the firm, since $v \geq \bar{u}$, this salary equals the expert's ex-post outside option and does not make it more difficult to satisfy the expert's capture-incentive compatibility constraint.

The maximum $w_{\emptyset}$ that is self-enforcing is found from making the firm's dynamic capture-incentive compatibility constraint bind:

$$
w_{\emptyset}^{M a x}=\bar{u}+\frac{\delta q(1-\eta) G}{1-\delta q \eta} .
$$

If the concealment cost is sufficiently high, that is, $\underline{c}+\bar{u} \geq w_{\emptyset}^{\text {Max }}$, deterring capture is costless. This condition can be rewritten as:

$$
\delta<\tilde{\delta} \equiv \frac{\underline{c}}{q(1-\eta) G+q \eta \underline{c}} .
$$


Now, suppose that capture is an issue because $\delta \geq \tilde{\delta}$. The principal must decide whether to deter or tolerate regulatory capture. If the principal does not prevent capture, she does not reward experts, namely, $\beta_{U}=\beta_{\emptyset}=0$, and welfare is

$$
W^{R D \text { tolerate }}=(1-q \eta) G+v-q(1-\eta)(D+\underline{c}) .
$$

To understand why, notice that the firm expects to get $(1-q \eta)(G-\underline{c})+v-\bar{u}$, where $w_{\emptyset}=\underline{c}+\bar{u}$ is the minimum salary that can induce an expert to conceal evidence. An expert expects to obtain $(1-q) \underline{c}+\bar{u}$. The expert obtains a rent when he does not manipulate evidence to make an empty report, that is, when $s=\emptyset$. While the salary is merely a transfer from the firm to the experts, the concealment cost enters the welfare expression whenever evidence is concealed. Note also that if $\eta \rightarrow 0$, it might be optimal for the principal to shut down the regulatory agency as the experts do not improve the authorization outcome while bearing inefficient concealment costs. This is the case when experts do not gain a lot of productivity at the regulatory agency, i.e., when $v$ is negligible.

Bonus policy. If the principal deters capture, she must create a wedge between the bonus the expert receives following a report that hurts the firm and the one following a report that is favorable: $\beta_{U}^{R D b o n u s}=w_{\emptyset}^{M a x}-\underline{c}-\bar{u}=\frac{\delta q(1-\eta) G}{1-\delta q \eta}-\underline{c}$ and $\beta_{\emptyset}=0$, and welfare is:

$$
W^{R D b o n u s}=(1-q) G-\lambda q\left(\frac{\delta q(1-\eta) G}{1-\delta q \eta}-\underline{c}\right)+v .
$$

Deterring capture through the bonus policy restores authorization efficiency but comes at the cost of rewarding the experts whenever they report evidence that is unfavorable to the firm. The following proposition describes the principal's favorite solution.

Proposition 1. Suppose that the revolving door must be kept open.

(a) If $\delta<\tilde{\delta}$, regulatory capture is not an issue and the principal sets $\beta_{U}=\beta_{\emptyset}=0$;

(b) if $\delta \geq \tilde{\delta}$, regulatory capture is an issue and the principal deters capture by setting $\beta_{U}^{R \text { Dbonus }}=w_{\emptyset}^{\text {Max }}-\underline{c}-\bar{u}$ and $\beta_{\emptyset}^{R D b o n u s}=0$ if and only if:

$$
\lambda \leq \hat{\lambda} \equiv \frac{(1-\eta)(1-\delta q \eta)(D-G+\underline{c})}{\delta q(1-\eta) G-(1-\delta q \eta) \underline{c}}
$$

where $\hat{\lambda}$ is decreasing in $\delta$. Otherwise, the principal does not prevent capture and sets $\beta_{U}=\beta_{\emptyset}=0$. 
When the discount factor is low, regulatory capture is not a problem as the maximum salary the firm can credibly promise to offer is lower than the concealment cost. For $\delta$ high enough, the implicit contract could be self-enforcing and this inevitably reduces welfare. The principal may decide to prevent capture by offering monetary incentives to the experts. The principal may refrain from doing so when this is more costly, i.e., when $\lambda$ is sufficiently high. Therefore, when paying high-powered incentives to experts faces more political opposition and/or creates more inefficiencies, regulatory capture is more likely to occur. The threshold value of $\lambda$ above which the revolving-door implicit contract is tolerated is decreasing in $\delta$. To understand why, notice that the maximum salary that the firm can credibly promise to pay to the experts is increasing in the discount factor, and so is the bonus that the principal would have to pay to an expert that makes an unfavorable report to discourage capture. In Figure 1, we graphically illustrate the parameter regions where the three equilibria arise, depending on the values of $\lambda$ and $\delta .{ }^{9}$

Also note that regulatory capture becomes more of an issue if $\eta$, i.e., the probability of a high concealment cost, decreases. Moreover, unless $\underline{c}$ is large, a decrease in $\eta$ increases $\hat{\lambda}$, which increases the parameter range under which the principal deters capture by providing a bonus. Thus, experts tend to benefit from a lower $\eta$ both directly and indirectly.

\subsection{Should We Close the Revolving Door?}

One policy option that we have so far deliberately overlooked is that of closing the revolving door. Clearly, this solution prevents regulatory capture in our set-up, as the firm would be unable to reward an accommodating expert. Yet, this solution is not without costs if experts may make a genuinely valuable contribution to the firms they join. In this subsection, we explore this trade-off more formally and put forward a novel option that can alleviate the welfare costs associated with closing the revolving door while retaining its benefit of deterring capture. Throughout, we will make the assumption that closing the revolving door means that an expert is forbidden from joining any firm operating in

\footnotetext{
${ }^{9}$ The figure is drawn using the following parameter values: $q=0.5, \eta=0.8, \underline{c}=0.5, G=20$, and $D=30$.
} 


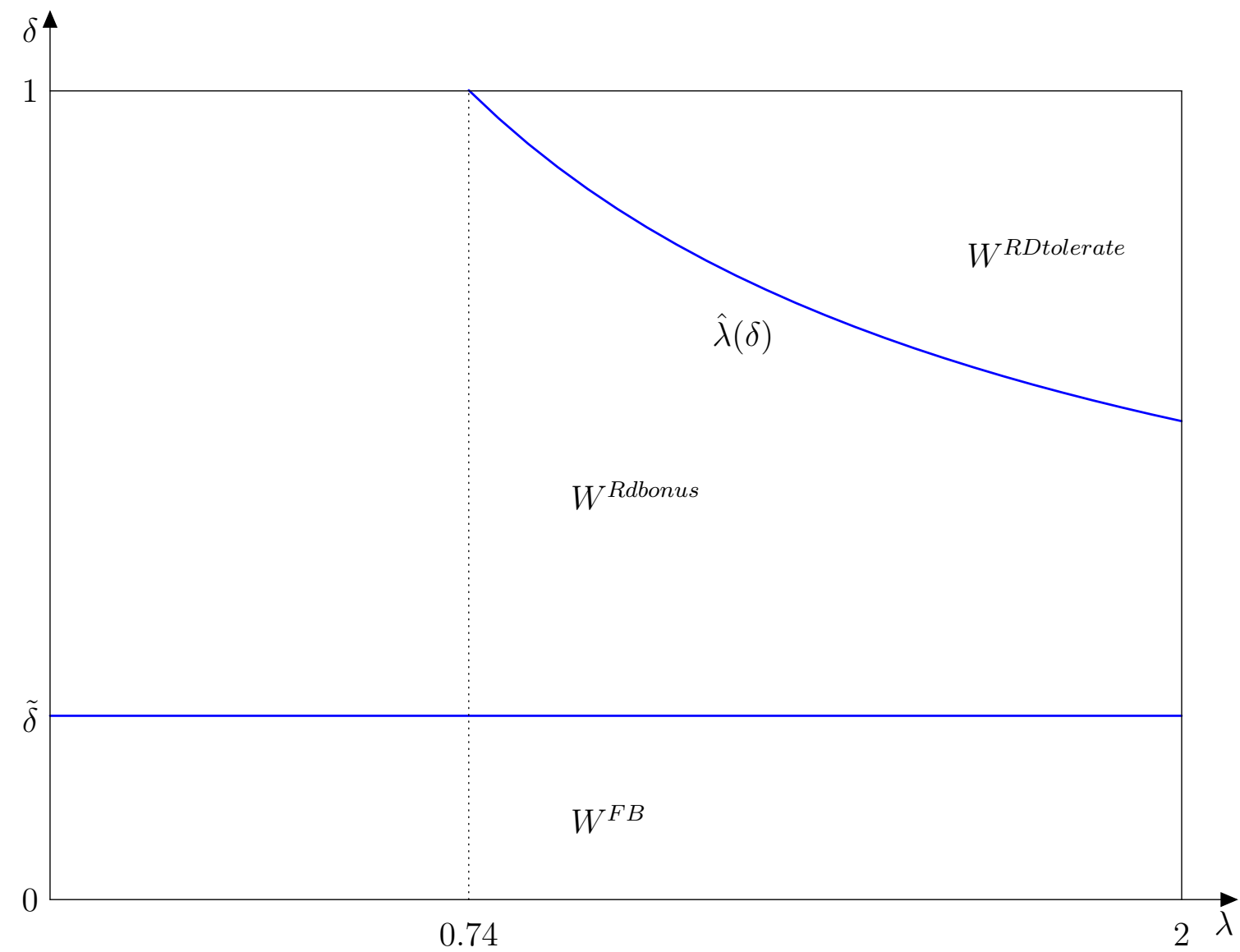

Figure 1: Welfare under optimal policies for different $\lambda$ and $\delta$ when the principal cannot close the revolving door.

the industry. ${ }^{10}$

Blanket ban. If the principal bans experts from joining the industry once they leave the regulatory agency, there is no need to offer rewards to the experts to induce truthful reporting. Welfare would be:

$$
W^{R \text { Dblanketban }}=(1-q) G \text {. }
$$

Like the high-reward option, closing the revolving door restores authorization efficiency. However, this policy entails the social cost associated with forgoing the experts' contri${ }^{10}$ To be truly effective, closing the revolving door should prohibit former experts from receiving any
direct or indirect compensation from firms in the regulated industry - not just the ones the expert directly
interacted with. Otherwise, experts may join or set up law firms, think tanks, or consulting firms and still
receive payments from previously regulated firms as reward for their friendliness. Coalitions of firms may
also take turns in hiring each other's (regulatory) experts to avoid more lenient employment restrictions. 
bution to the industry.

Conditional ban. An alternative option the principal should entertain is that of opening the revolving door conditionally on the information an expert reveals in his report. Specifically, suppose that only an expert who reports $r=U$ can later take up positions in the industry and is otherwise banned from doing so. This induces an expert who has obtained unfavorable information to reveal it, thereby preventing capture: a firm cannot promise a future job to an accommodating expert, since the expert can later be hired only if his report does not benefit the firm. This solution would ensure authorization efficiency while alleviating the ex-post welfare cost that employment restrictions entail. Welfare under this solution would be:

$$
W^{R D \text { conditionalban }}=(1-q) G+q v
$$

which is strictly higher than $W^{R D b l a n k e t b a n}$. If the principal wants to deter regulatory capture, she will prefer selectively closing the revolving door to keeping it open and relying on the bonus policy if and only if:

$$
v<\hat{v} \equiv \lambda \frac{q}{1-q} \underbrace{\left(\frac{\delta q(1-\eta) G}{1-\delta q \eta}-\underline{c}\right)}_{\beta_{U}^{\text {RDbonus }}} .
$$

All factors which inflate the capture-preventing bonus, such as a lower concealment cost $\underline{c}$, a higher probability that information can be concealed $1-\eta$, a higher profit associated with authorization $G$, a higher likelihood that the state is unsafe $q$, and a higher discount factor $\delta$ make a conditional ban more appealing. By contrast, a larger expert's contribution to the firm $v$ and a lower inefficiency caused by paying a high salary to the expert $\lambda$ make the principal lean towards using bonuses to deter capture and keeping the revolving door always open. Furthermore, selectively closing the revolving door strictly dominates tolerating capture when

$$
v \leq \tilde{v} \equiv \frac{q}{1-q}(1-\eta)(D-G+\underline{c})
$$

Note that $\tilde{v}$ coincides with $\hat{v}$ when $\lambda=\hat{\lambda}$. Since $\hat{v}$ is increasing in $\lambda$, the relevant condition for the conditional ban to be the preferred policy is $v<\hat{v}$ when $\lambda \leq \hat{\lambda}$ and $v<\tilde{v}$ when $\lambda>\hat{\lambda}$. 
The following proposition pins down the principal's favorite solution when regulatory capture is an issue. It is straightforward to show that keeping the revolving door open is socially beneficial if the interaction is sporadic (i.e., in the one-shot game) or the players are not sufficiently patient $(\delta<\tilde{\delta})$ so that regulatory capture does not represent a concern. ${ }^{11}$

Proposition 2. Suppose that $\delta \geq \tilde{\delta}$.

1. If $\lambda \leq \hat{\lambda}$, the principal prevents regulatory capture by

(a) selectively closing the revolving door if $v<\hat{v}$;

(b) using the bonus policy if $v \geq \hat{v}$.

2. If $\lambda>\hat{\lambda}$, the principal

(a) prevents capture by selectively closing the revolving door if $v \leq \tilde{v}$;

(b) tolerates capture if $v>\tilde{v}$.

Deterring capture is worthwhile when the damages that an unsafe activity can give rise to are substantial relative to the gains of prohibiting production. We have shown different alternatives the principal should entertain when fighting capture. This can be achieved directly by rewarding the expert who reports unfavorable evidence with a monetary bonus. In this way, the expert acts as a bounty hunter, looking for bad evidence. Capture can also be prevented indirectly, by prohibiting the firm from using the revolving door to reward a friendly expert. This does not necessarily imply that experts cannot join the private sector under any circumstances. In fact, we have shown that the principal should leave the door open for those experts who reveal evidence that does not benefit the regulated firm. In that case, it would be difficult to argue that the firm's recruitment of the expert is part of a shady do ut des scheme.

Figure 2 illustrates the regions of parameter values where the different policy options are optimally chosen by the principal as a function of $v$ and $\delta .^{12}$ A conditional ban

\footnotetext{
${ }^{11}$ See Proposition 7 in the appendix for the analog of Proposition 2 for the case in which the signal $s$ is not always informative when $\theta=U$.

${ }^{12}$ The figure is drawn using the following parameter values: $q=0.5, \eta=0.8, \underline{c}=0.5, G=20, D=30$, and $\lambda=1$. Further note that $\hat{\delta}$ is derived from $\hat{\lambda}$ when the above values for the parameters are used.
} 
is preferred when $\delta$ is relatively large as compared to $v$. Intuitively, by increasing the monetary reward needed to deter capture, a higher $\delta$ reduces the attractiveness of the bonus scheme. This suggests that the usefulness of the conditional ban is greater in industries where there is frequent firm-regulatory agency interaction. All the more so when the value of the regulatory expertise $v$ is not particularly large.

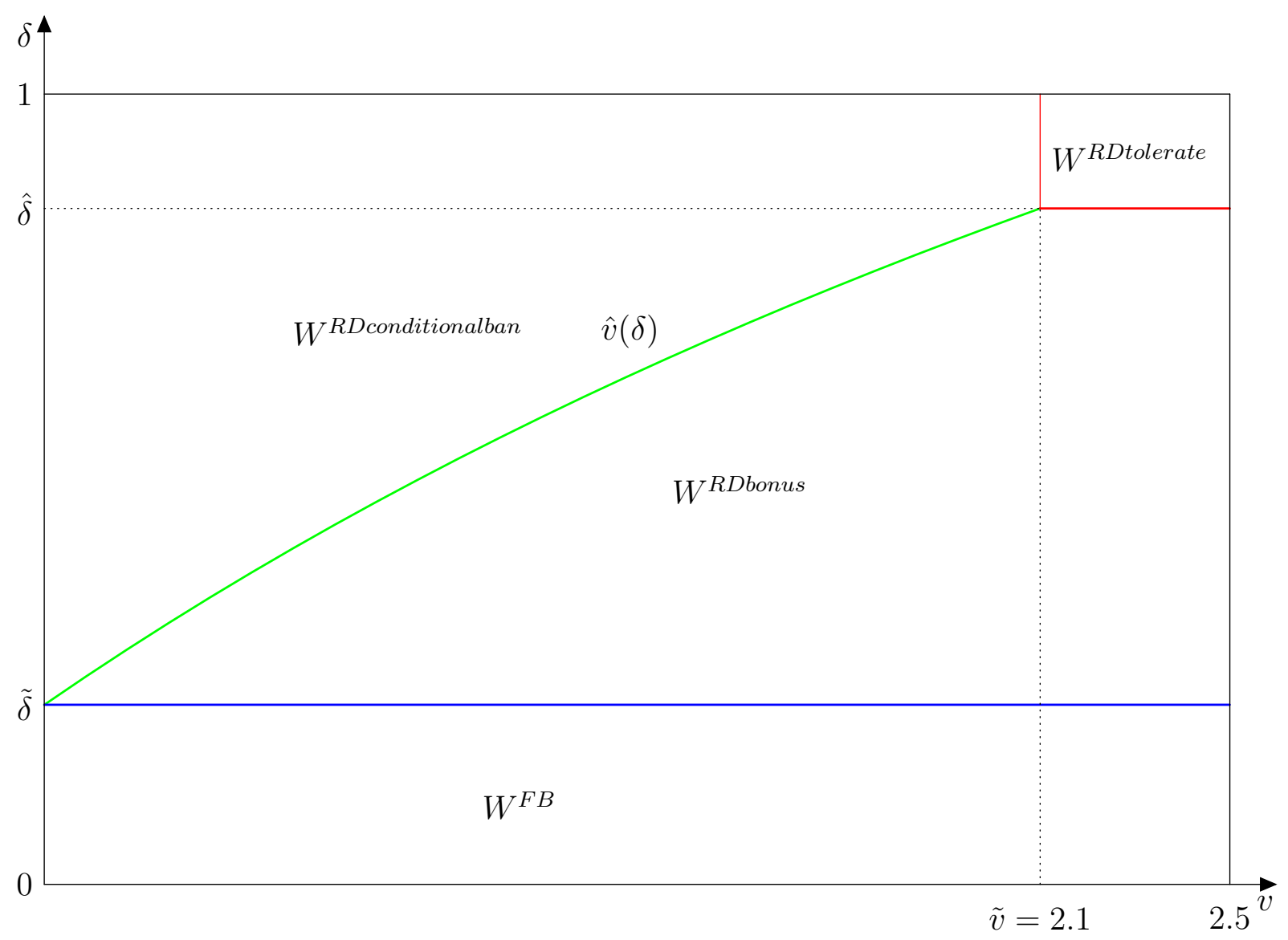

Figure 2: Welfare under optimal polices for different $v$ and $\delta$ when the principal can close the revolving door.

\subsection{Revolving Door versus Implicit Bribes}

In this subsection, we aim to shed light on the critical role played by the revolving door in sustaining regulatory capture as an equilibrium phenomenon. To this end, suppose that the firm implicitly promises to pay a bribe $b$ to an expert who reports $r=\emptyset$. While, at first blush, this payment looks strikingly similar to the wage, there is a crucial difference: 
being illegal, the bribe cannot be made public. As a result, only the expert who receives the bribe and the firm observe whether it has been paid or not. Consider a bribery implicit contract akin to the revolving-door implicit contract described earlier, with the difference that a bribe is paid instead of a post-agency wage. We obtain the following result.

Proposition 3. There does not exist a self-enforcing bribery implicit contract which induces the experts to conceal evidence.

The reason why the implicit promise of a bribe cannot induce the experts to conceal evidence lies in the private nature of this illegal payment. As the period $t+l$ expert, with $l=1,2, \ldots$ observes the report sent by the period $t$ expert but not whether or not he received the promised bribe, let alone its size, the period $t+l$ expert has no way of knowing whether the firm stands by its promises. As bribing an expert would not affect future experts' beliefs and behavior, the firm does not have an incentive to follow through on the promised payment. Hence, no positive bribe can credibly be promised.

This result is robust to contemplating a different timing of the bribe and, with some qualifications, to allowing experts to exchange messages. As for the first point, suppose that the firm pays the bribe before the expert sends the report. In this case, the expert who has already pocketed the bribe lacks the incentive to manipulate evidence whenever $\Delta_{\beta} \equiv \beta_{U}-\beta_{\emptyset} \geq 0$. Stated differently, it is the expert's capture-incentive compatibility constraint that would not be satisfied: no matter what he reports, this does not affect the payment he receives from the firm. Hence, the expert would only take into account the bonus differential and the manipulation cost in choosing his report. If $\Delta_{\beta}=0$ and $\underline{c}$ is strictly positive, reporting truthfully the collected evidence would be strictly dominant.

As for the experts' ability to exchange messages, this could be used to coordinate punishments, facilitating capture by ensuring that a firm that deviates in a given period is punished in the continuation game. We obtain the following remark.

Remark 1. Suppose that, in each period $t$, the period $t$ expert can send a message $m_{t}$ that is observed by all future experts. Then, the bribery implicit contract may be self-enforcing.

Note that it is not necessary that the message can be observed by all future experts: if only the next period's expert can observe each expert's message, a similar strategy as above works, in which the message sent also depends on the previous expert's message. 
There are some caveats to this logic, though. Firstly, if experts could commit to messages before sending the report to the principal, the regulatory capture equilibrium could unravel. This is because the experts could blackmail the firm, fully extracting the firm's surplus under the threat of spreading information that the firm deviated, as formally shown in a different setting by Barron and Guo (2021), to which we refer the interested reader.

Secondly, it must be that the principal cannot observe or at least cannot verify the messages that experts exchange. Otherwise, the principal could have proof that corruption has occurred and could then take some appropriate measures to tackle it. Unlike the bribe, offering a reward to an expert in the form of a well-paid job gives the firm a justification that can hold up in court: the firm could always argue that it has hired the expert for his skills and expertise and not to reward his possibly lenient behavior during his term at the regulatory agency. In practice, this would make it harder for the principal to condition her regulatory decision on this observation. While the firm could increase the bribe, so as to make up for the risk of being caught by the principal, such a bribery implicit contract, though self-enforcing, would not exactly replicate the equilibrium outcome described for the revolving door. It would be more costly to implement for the firm, which would thus prefer the revolving-door equilibrium.

That being said, when the revolving door is closed, a firm may resort to bribes to capture experts. An implication of Remark 1 is that what types of bribes are used depends on how authorities react to observing potential signals of bribes. If a visible bribe, such as an expert driving a fancy car he could hardly afford given his wage, triggers an investigation, bribes would likely be hidden. However, if authorities would not act upon such signals, a firm would choose very visible bribes in order to show everyone that it keeps its promises.

This subsection has allowed us to highlight that bribes cannot substitute for the revolving door as a tool to sustain regulatory capture as an equilibrium phenomenon. The public nature of the recruitment decision enables the firm to signal its eagerness to reward friendly experts and acts as a commitment device. Being publicly observed, the firm has a powerful incentive to make good on its implicit promise. Importantly, the capture mechanism we have described works in a way that is not typically emphasized by academics, 
policy makers, and observers: a firm that provides friendly experts with lucrative job opportunities may be driven by the desire to signal to future experts that they could earn rents by being accommodating rather than by an inclination to positively reciprocate past favors. Then, the existence of future rents is crucial for regulatory capture to occur.

\subsection{Discussion}

In this subsection, we discuss the role of some of our key assumptions and briefly explore the scope of the chief policy prescriptions arising out of our analysis.

Extortion. In the model, we have suggested that the revolving door should be kept open depending on the evidence that the expert reveals. If an expert makes a report that cannot be deemed friendly to the regulated firm, the occurrence of capture can be excluded and, as a result, there is no reason to prohibit the expert from joining the private sector after leaving the agency. A possible limitation to this suggested solution arises if an expert could forge evidence that harms the regulated firm. If so, both a reward policy that sets $\beta_{U}>\beta_{\emptyset}$ and the conditional ban might backfire and may result in framing and extortion (e.g., Khalil et al., 2010 show that a reward to prevent collusion can lead to extortion by making the threat of framing credible). To avoid this undesirable outcome, an expert's damning report should be based on objective evidence that could be reviewed by other experts or hold up in a court of law, if the firm decides to appeal the unfavorable decision and turn to the judicial authority. Indeed, provisions to avoid an unfairly or unjust treatment of applicants are in place in both the U.S. and Europe. There, patent applicants and drugmakers seeking marketing authorization whose applications have been rejected can request the evidence to be reviewed by different experts. ${ }^{13}$ Such provisions substantially diminish the expert's framing ability.

\footnotetext{
${ }^{13}$ For instance, in the U.S. an applicant for a patent can appeal to the Patent Trial and Appeal Board if their claims have been twice rejected (U.S. Code, Title 35, Section 134). In Europe, Part VI of the Convention on the Grant of European Patents establishes the appeals procedure. As for the evaluation of medicines, the European Medicines Agency provides a step-by-step procedure for marketing authorization applications that include the right for the developer to appeal an unfavorable decision, in which case new experts are appointed to evaluate the available evidence.
} 
Partially-verifiable reports. In our model, we have assumed that damages are nonverifiable and, as a result, the principal does not know whether a favorable report was issued because production was indeed safe or because evidence of its unsafety had been concealed. As said, a remarkable feature of the revolving-door equilibrium is that experts-firm collusion can take place without leaving a smoking gun behind, rendering many standard anti-corruption policies ineffective. However, with some delay, the principal may learn whether production authorized in a given period hurt someone. For instance, depending on the activity, there might emerge evidence of local contamination, anti-competitive behavior, or other types of non-compliance with regulations or there might be accidents. When this happens, the principal may revoke the incorrectly-given authorization. This does not affect the general thrust of our analysis, although it may reduce the appeal of policies which involve the closure of the revolving door.

To see this, suppose that with some positive probability the principal learns the true $\theta$ and, if $r=\emptyset$ when $\theta=U$, the firm and/or the expert who filled the inaccurate report are punished. For instance, the firm may lose $G$ and the expert may be fined and/or his reputation tarnished. Ex-post detection of the true state of the world makes it more complicated to sustain capture. This is because the firm's expected profit from engaging in regulatory capture is diminished (e.g., if the authorization may be revoked) and the expert is more reluctant to conceal unfavorable evidence (if his reputation may take a hit and his wallet lightened). There can also be a complementarity between running investigations of suspiciously favorable reports and the use of bonuses to prevent regulatory capture.

\section{Extensions}

In what follows we extend the baseline model in several directions. All proofs and most technical details are provided in Appendix B.

\subsection{Firm Able to Observe Signal or State}

What if the firm also observes the same signal as the expert or knows the (current) state of the world? If the firm does not observe the signal and just knows, but cannot condition its wage on, the state of the world, our analysis does not change. We assume that the 
firm is not able to make the wage dependent on the state of the world. This assumption seems plausible in a real-world setting in which damages might be rare and only revealed in the long-run, even if $\theta=U$.

Remark 2. If the firm knows the state of the world or observes the expert's signal, Propositions 1 and 2 apply.

Note that if there are different types of experts, as in Subsection 4.4, and types are not observable, the firm learns something about the expert's type when it observes the expert's signal. This makes it harder to commit to hire an expert that did not find unfavorable evidence, but does not change our results qualitatively. ${ }^{14}$

\subsection{Effort Necessary for Expert to Observe Signal}

So far, we have assumed that experts automatically observe if $\theta=U$. We now analyze the case in which an expert must make an effort to learn whether the state is unsafe. Specifically, before learning his concealment cost $c$, the expert privately chooses $e \in\{0,1\}$ at cost $c_{e} e$. If he does not exert effort $(e=0)$, the signal is always uninformative. If he exerts effort $(e=1)$, the signal is informative. If the principal wants to implement $e=1$, she needs to motivate the expert sufficiently. If there are no employment restrictions, this constraint is: ${ }^{15}$

$$
\frac{c_{e}}{q} \leq \eta\left[\bar{u}+\beta_{U}\right]+(1-\eta)\left[\max \left\{\bar{u}+\beta_{U}, \max \left\{w_{\emptyset}, \bar{u}\right\}+\beta_{\emptyset}-\underline{c}\right\}\right]
$$

Naturally, the principal would not want to implement $e=1$, if the unsafe state is very unlikely, the effort cost is very high, or she tolerates capture anyway. The principal can provide incentives to acquire information via $\beta_{U}$ or let them be provided by future wages. Remarkably, the conditional ban presents the additional advantage of motivating information acquisition, as an expert may look for (unfavorable) evidence so as to get access to lucrative job opportunities in the industry thereafter.

Welfare is not directly comparable to the previous subsections, of course. Either the principal induces $e=1$, in which case effort costs occur; or the principal does not do so,

\footnotetext{
${ }^{14}$ Also see Subsection 5.3 of an earlier version of this paper, De Chiara and Schwarz (2020).

${ }^{15}$ If there are employment restrictions, the according wages become zero.
} 
in which case there are not only no effort costs but also no concealment costs for experts who do not report unfavorable evidence.

The following proposition pins down the principal's favorite solution for the case in which the expert has an effort cost for acquiring information. Preventing capture only by means of a reward scheme may be more expensive in that case. This makes the policy of opening the revolving door conditional on an unfavorable report more desirable.

Proposition 4. Suppose an expert has to exert effort to potentially observe an informative signal. If

$$
\begin{array}{r}
q G+c_{e}>q D-\max \left\{(1-q) v+\lambda q \beta_{U E f f o r t}^{R D \text { condionalban }}, \lambda q \beta_{U E \text { ffort }}^{R D \text { deter }}\right\} \\
\text { where } \beta_{U E f \text { fort }}^{\text {RDdeter }}=\max \left\{\frac{c_{e}}{q}, \frac{\delta q(1-\eta) G}{1-\delta q \eta}-\underline{c}, 0\right\} \geq \beta_{U}^{\text {RDdeter }}, \\
\text { and } \beta_{U E \text { fonditionalban }}^{R D \text { cond }}=\max \left\{c_{e} / q-\bar{u}, 0\right\},
\end{array}
$$

then the principal tolerates regulatory capture. By contrast, if (7) does not hold, the principal deters capture with a high bonus if $v>\lambda \frac{q}{1-q}\left(\beta_{U E f \text { fort }}^{R D d e r}-\beta_{U E f \text { cond }}^{R D \text { condionalban }}\right)$ and by selectively closing the revolving door otherwise.

\subsection{Moral Hazard on Safety Care}

The firm may take actions that affect the probability that the state of the world is safe. For example, in the case of environmental regulation, the firm can devote resources to comply with the existing laws and rules. In the appendix, we develop an extension to the baseline model in which we endogenize the state of the world. This extension makes our model similar to the work on moral hazard on safety care (e.g., Hiriart et al., 2010) or crime prevention (e.g., Ortner and Chassang, 2018) where, in addition to inducing truthful reporting, the principal wants to prompt the firm to take the desired action. We find that the conditional ban may give rise to perverse incentives as the firm may underinvest in safety so as to increase its chances of being able to hire an expert afterward. This shortcoming is more likely to arise when the specific knowledge that the expert could bring to the firm, $v-\bar{u}$, and the compliance cost are relatively large as compared to the benefits generated by a positive report $G$. By contrast, the bonus policy does not suffer from this downside and can provide optimal incentives to invest and deter capture, although its convenience decreases in the compliance cost. 


\subsection{Experts versus Bureaucrats}

Suppose that the principal can assign the task of collecting information about the state $\theta$ to a different type of officials, that we henceforth call bureaucrats. Unlike experts, bureaucrats may fail to observe that the state is unsafe and the value of their expertise to the private sector may be lower. In particular, let us assume that a bureaucrat observes $s=U$ when $\theta=U$ with probability $\epsilon<1$. Therefore, with probability $q \epsilon$ a bureaucrat observes $s=U$, whereas with complementary probability $1-q \epsilon$, he observes $s=\emptyset$. Moreover, by joining the private sector at the end of his term at the agency, a bureaucrat can increase the profit of the regulated firm and that of other organizations by $v_{B}$ and $\bar{u}_{B}$, respectively, with $v_{B} \geq \bar{u}_{B} \geq 0, v_{B} \leq v$, and $\bar{u}_{B} \leq \bar{u}$. In each period, the principal decides whether to hire a bureaucrat or an expert and their type is publicly observable. In practice, regulatory agencies may assign specialized experts to monitor the firm or, alternatively, they may rely on employees who have a less profound understanding of the industry. Because of observability, there is no reason to hire a mix of experts and bureaucrats.

As we show in the appendix, the principal may decide to employ bureaucrats rather than experts in two distinct, though related, cases. Firstly, for intermediate values of $\delta$, capture is an issue if the principal employs experts, but not if she employs bureaucrats. This is because the firm may be more reluctant to engage in capture if there is already a high probability that the officials will not collect unfavorable information. In that region, it is desirable to employ bureaucrats unless the efficiency loss due to their employment is too sizable. Secondly, when capture is prevented via the bonus policy, the principal may prefer to employ bureaucrats to save on the total wage bill: the bonus needed to deter capture as well as the probability of paying it is lower when the signal is collected by bureaucrats instead of experts. By contrast, the principal always prefers to hire experts when capture is tolerated or when capture is deterred by selectively closing the revolving door. Employing bureaucrats would only magnify the welfare losses (i.e., the reduction in the value added of the officials to the private sector and the probability that an unsafe product is authorized) with no offsetting benefit in return.

By contrast, when neither the firm nor the principal knows the official's type, e.g., when the monitoring task is assigned to a new recruit. Then, regulatory capture is more 
difficult to implement when the official's ability to find evidence unfavorable to the firm is positively correlated with the value of the official's regulatory expertise to the industry. Intuitively, concealing information worsens the official's job prospect as he foregoes the opportunity to signal his ability to the industry. ${ }^{16}$

\subsection{Cooling-off Periods}

In this subsection, we examine how post-employment restrictions affect the results of our analysis. In many countries, after leaving governmental agencies, experts are temporarily prohibited from joining firms or organizations in the industry they used to regulate. ${ }^{17}$ An implication of such restrictions, which are also known as cooling-off periods, is that firms' and experts' benefits from employment are delayed. To account for this effect in our set-up, we now assume that the payoffs that the firm (or other industry players) and the expert can obtain from the employment relationship in stage 3 are evaluated by the factor $\frac{1}{1+\gamma}$ in stage 2 . The term $\gamma \in[0, \infty)$ reflects the employment restrictions, which is the principal's choice variable. Thus far, we have confined attention to two polar policies: that in which an expert can freely join other firms or organizations once his term at the regulatory agency is over (i.e., $\gamma=0$ ), and that in which an expert is banned from joining the industry after leaving the agency $(\gamma=\infty)$.

If the principal sets out to thwart capture, besides the conditional ban and the bonus policy, she may decide to set $\gamma$ high enough, and can use this policy tool in combination with the reward scheme. In the same fashion as the conditional ban, the principal could also contemplate making the length of the cooling-off period conditional on the expert's report. When considering this option, we will assume that an expert can join the industry without delay if $r=U$, whereas there might be a cooling-off period if $r=\emptyset$.

Employment restrictions should not be imposed if interaction is one-off, as they would only reduce welfare. Notice that $W_{\text {cool }}^{S G}=(1-q) G+q \mathbb{1}_{\text {select }} v+\frac{\left(1-q \mathbb{1}_{\text {select }}\right) v}{1+\gamma}$, where $\mathbb{1}_{\text {select }}$ is an indicator function that takes value 1 if the cooling-off period is conditional on $r=\emptyset$. It is immediate to see that this welfare expression is decreasing in $\gamma$. Recall that capture requires an explicit agreement when regulatory interaction is infrequent. As such, it is

\footnotetext{
${ }^{16}$ Also see an earlier version of this paper, De Chiara and Schwarz (2020).

${ }^{17}$ We provide a summary of the most important employment restrictions in the U.S. and Europe in Appendix B.5.
} 
better prevented by other anti-corruption policies which rely on seeking out some hard evidence that can be used as a smoking gun.

Consider frequently repeated interaction now. The firm has the opportunity to renege on the implicit understanding with the expert once the cooling-off period is over. As a result, the expression for the firm's dynamic capture-incentive compatibility constraint is unchanged:

$$
(1-\delta)\left[v-w_{\emptyset}\right]+\delta \pi_{\text {cool }}^{R D} \geq(1-\delta)(v-\bar{u})+\delta \pi_{\text {cool }}^{S G}
$$

Conversely, the expert's capture incentive compatibility constraint must be amended. At the time he can conceal evidence, the expert anticipates that he will not be able to immediately join the industry after leaving the agency:

$$
\frac{1}{1+\gamma} \max \left\{w_{\emptyset}, \bar{u}\right\}+\beta_{\emptyset}-\underline{c} \geq\left(\frac{1}{1+\gamma}+\mathbb{1}_{\text {select }} \frac{\gamma}{1+\gamma}\right) \max \left\{w_{U}, \bar{u}\right\}+\beta_{U} .
$$

If the principal tolerates capture, $\gamma=0$. Intuitively, there is no point in delaying the time at which the firm can hire the expert if capture is not prevented. If capture is prevented, the principal chooses $\gamma$ to maximize welfare:

$$
W_{\text {cool }}^{R D \text { deter }}=(1-q) G+q \mathbb{1}_{\text {select }} v+\frac{\left(1-q \mathbb{1}_{\text {select }}\right) v}{1+\gamma}-\lambda q \beta_{U}^{R D d e t e r}(\gamma) .
$$

By inspecting the welfare expression, we can already conclude when the principal may want to use the cooling-off period.

Remark 3. The principal may use the cooling-off period only to reduce the social cost of the bonus scheme.

It is immediate to see that if $\beta_{U}^{R D d e t e r}=0$, for instance, because $\underline{c}$ is high, an increase in $\gamma$ would decrease welfare exactly as in the case when there is no repeated interaction. By itself, a finite cooling-off period does not affect the firm's willingness to make good on the promise to hire the accommodating expert. Thus, the only reason why the principal may want to use this type of post-agency employment restriction is to reduce the bonus she has to pay to an expert who reports $r=U$. The following proposition illustrates how the cooling-off period should be used.

Proposition 5. When the cooling-off period is used, it is always efficient to condition its length on the expert's report. The optimal cooling-off period is increasing in $G$ and $\gamma$, whereas its relation with $v$ and $\bar{u}$ is ambiguous. 
The first striking result is that cooling-off periods should always be contingent on the expert's report and the reason is threefold. First, as an expert makes a valuable contribution to the industry, it is desirable that the recourse to this employment restriction be as limited as possible. Second, since the firm profits from employing the expert and inducing concealment of unfavorable evidence kicks in the employment restriction, the firm is less willing to engage in regulatory capture and, consequently, it can be deterred more easily. Third, the opportunity cost of concealing unfavorable information is higher for the expert, thereby lowering the bonus needed to induce truthful reporting.

When used, the length of the cooling-off period positively depends on $G$ and $\lambda$ as these parameters can increase the benefit of reducing the social cost of paying a bonus. When the value of the regulatory expertise $v$ is higher, longer cooling-off periods have a direct negative impact on welfare. However, a lower bonus is needed to deter regulatory capture since this is less appealing to the firm, which benefits more from employing an expert. Therefore, a higher $v$ has an ambiguous effect on cooling-off periods. ${ }^{18}$ Because the optimal length of the cooling-off period depends on various factors, it seems unlikely that a uniform cooling-off period across regulatory agencies is optimal.

Also note that an expert always suffers from a higher $\gamma$ because his outside option, to which he is held, will decrease as he could take up a job in which his prior expertise is valuable only later. Similarly, the firm's profit will decrease because it can only employ the experts later. If the cooling-off period is very long, these effects will outweigh the efficiency improvements due to a lower bonus payment. This is so even without assuming any deterioration in the value of the regulatory expertise due to the inability of working for the industry for some time. Thus, a poorly-chosen length of a cooling-off period can make everyone worse off.

\subsection{Multiple per-period reports}

In this subsection, we examine the design of capture-prevention tools in a setting in which each expert interacts several times with the regulated firm. In particular, we focus on how to adapt our suggested policy of conditionally-opening the revolving door in this more

\footnotetext{
${ }^{18} \mathrm{~A}$ higher $\bar{u}$ has an ambiguous effect on the equilibrium level of $\gamma$ too, but this is because $\bar{u}$ has an uncertain effect on the equilibrium bonus.
} 
complex environment. To keep the model tractable, and yet provide valuable insights, we assume that each expert is asked to make two reports on two independent signals associated with the safety of the firm's activity. In practice, one could think of a firm applying for two distinct licences or patents to the agency, or complying with two distinct pieces of regulation. Below, we provide further details about the set-up and we illustrate the main results that we obtain, while we relegate an extensive analysis of this environment to Appendix B.6.

We assume that, in each period $t$, the firm independently draws two binary random variables $\theta_{t}^{1}$ and $\theta_{t}^{2}$ from the same probability distribution on $\{S, U\}$, with respective probabilities $(1-q, q)$. The expert sequentially observes two independent signals $s_{t}^{i}$, with $i=1,2$ that reveal whether $\theta_{t}^{i}=U$ and makes a report to the principal. With probability $1-\eta$, the true signal can be concealed. Each expert makes the report on $s_{t}^{1}$ before observing $s_{t}^{2}$. There is no within-period discounting. Any time a firm's activity is authorized, the firm obtains $G$ and, if the activity is unsafe, there are damages $D$ to third parties. Only after having collected and reported on the two signals, the expert can give a positive contribution to the industry. Specifically, he can yield $v$ to the regulated firm and $\bar{u}$ to other firms.

For this amended model, the timing of the stage game is as follows:

0. The principal commits to the authorization decision as a function of the report $x_{r} \in\{0,1\}$.

1. Nature draws the states of the world $\theta_{t}^{i}$ and the concealment $\operatorname{costs} c_{t}^{i}$ for $i=1,2$. The principal sets the bonus scheme for the experts, $\beta_{t}\left(r_{t}^{1}, r_{t}^{2}\right) \geq 0$.

2. If the expert accepts to work for the regulatory agency,

(a) he observes the signal $s_{t}^{1}$ and sends a public report $r_{t}^{1}$ to the principal who authorizes or not production according to $x_{r}$.

(b) Then, the expert observes the signal $s_{t}^{2}$ and sends a public report $r_{t}^{2}$ to the principal who authorizes or not production according to $x_{r}$ and pays the expert $\beta_{t}$.

3. The firm may make an offer $w_{t}$ to the expert, who can either accept this offer or take up his outside option. 
Consider the following revolving-door implicit contract with multiple per-period reports, denoted RD2: (i) experts always conceal evidence when the concealment cost is $c=\underline{c}$; (ii) the firm rewards experts who report $r^{1}=\emptyset$ and/or $r^{2}=\emptyset$ with improved job prospects; (iii) if at period $t+l$ the firm did not improve the job prospects of the expert who reported $r^{1}=\emptyset$ and/or $r^{2}=\emptyset$, then at $t+l^{\prime}$ with $l^{\prime}>l$, the experts always reveal unfavorable evidence.

We continue to restrict attention to stationary equilibria. The firm's offered salary $w_{r r}$ and the principal's bonus schedule $\beta_{r r}$ are both functions of the two reports made by an expert. To focus this extension on the design of policies that deter regulatory capture, we now impose the following assumption that will be maintained throughout the subsection.

$$
\frac{q}{1-q}(1-\eta)(D+\underline{c}-G)>v
$$

The above inequality implies that closing the revolving door following a report that benefits the firm is preferred to tolerating capture from a social standpoint, i.e., $(1-q) G+q v>$ $(1-q \eta) G-q(1-\eta)(D+\underline{c})+v$. It requires that the social cost of concealing that the state is bad, $D+\underline{c}-G$, be relatively large as compared to the expert's contribution to industry profits, $v$, and that the probabilities that the state is unsafe and evidence can be concealed be non-negligible. The above restriction dramatically reduces the number of cases we have to contemplate as we do not need to worry about the possibility that the principal may find it advantageous to partially tolerate capture. This is because we need not consider the possibility that the principal may allow the expert's concealment of unfavorable evidence in either stage 2.a or stage 2.b. At the same time, the restriction does not undermine our goal of more properly designing the revolving-door policy. ${ }^{19} \mathrm{We}$ leave aside the possibility that the agency may employ distinct experts to collect the two signals, which is a question that has received considerable attention in the literature (e.g., see Laffont and Martimort, 1999). ${ }^{20}$ We also assume that the expert must send $r_{t}^{1}$ before the end of stage 2.a, for otherwise an approval does not yield $G$ to the firm, that is, the first activity must be timely approved to be profitable.

\footnotetext{
${ }^{19}$ Furthermore, note that if (9) does not hold, conditionally closing the revolving door is never socially beneficial and the principal's choice boils down to either tolerating capture or deterring it with bonuses.

${ }^{20}$ This restriction can be justified on the grounds that there might be shortages of trained personnel who can collect valuable information about $\theta$. In the real world, it may be impractical to assign to each expert only one report to make.
} 
To prevent capture, the principal could close the revolving door, irrespective of what reports an expert makes. Such a blanket ban on joining the industry would result in the following welfare:

$$
W^{R D 2 b l a n k e t b a n}=2(1-q) G
$$

because the firm would not be able to reward an accommodating expert and, as a consequence, capture would not occur. Our first result can straightforwardly be proved.

Remark 4. Unconditionally closing the revolving door is inefficient.

To prove this option, it is sufficient to show that it is dominated by opening the revolving door conditional on the expert's sending two unfavorable reports, i.e., $r^{1}=r^{2}=$ $U$ - option we refer to as $U U$. As an expert can be hired only if he is never accommodating, then the revolving door cannot give rise to regulatory capture. As the firm will leap at the chance of hiring a valuable expert if both reports have been unfavorable, welfare is:

$$
W_{U U}^{R D 2 \text { conditionalban }}=2(1-q) G+q^{2} v>W^{R D 2 \text { close }} .
$$

There are other anti-capture policies that the principal could pursue which involve a joint use of bonuses and selectively-closing the revolving door. Firstly, the principal could keep the revolving door open if the first report has been unfavorable - we refer to this alternative as $U r$, because the second report does not affect whether the expert can join the industry. Secondly, the principal could open the revolving door if the second report has been unfavorable - we label this alternative $r U$. While both options suffice to prevent full evidence manipulation, they are vulnerable to partial capture, whereby the firm promises to reward the expert if the second (respectively, the first) report is favorable. For this reason, the principal must promise a reward to the expert when the reports are unfavorable to the firm. The next result compares these two policies from the principal's perspective.

Remark 5. The principal always prefers Ur to $r U$.

To understand why, consider that with policy $r U$, if partial capture occurred, the expert would conceal an unfavorable signal in stage 2.a whenever the concealment cost is low. With policy $U r$, if partial capture were to occur, the expert would conceal an unfavorable signal in stage 2.b whenever the concealment cost is low and the first report 
was unfavorable. As the firm would get a higher expected gross profit with $r U$ than with $U r$ by inducing evidence concealment in one period, deterring partial capture is more expensive for the principal.

The principal could also open the revolving door as long as at least one report has been unfavorable (that is, irrespective of its order) or fully rely on bonuses to deter capture for both reports. We henceforth label these options $1 U$ and Bonus2, respectively. We obtain the following proposition by comparing the welfare expressions and performing comparative statics on two key parameters, $\lambda$ and $v$.

Remark 6. When each expert collects and reports on two signals sequentially, the principal will deter capture by choosing a policy $P \in\{U U, U r, 1 U$, Bonus2 $\}$. Moreover,

(a) an increase in $v$ reduces the appeal of $U U$ and makes $1 U$ relatively better than $U r$. For $v$ sufficiently high, Bonus2 is the unique optimal policy.

(b) An increase in $\lambda$ reduces the appeal of Bonus 2 and makes Ur relatively better than $1 U$. For $\lambda$ sufficiently high, $U U$ is the unique optimal policy.

When the value of regulatory expertise $v$ is more significant, closing the revolving door is less desirable and the principal leans towards the use of bonuses to prevent capture. Among the policies which involve selectively closing the revolving door, the principal prefers to use $1 U$ than $U r$ because the former deters capture while leaving a larger chance for the expert to join the private sector. When paying the expert is more expensive for the principal, i.e., $\lambda$ is higher, selectively closing the revolving door becomes a more attractive option than using bonuses. If $\lambda$ is very high relative to $v$, the principal would optimally resort to $U U$, namely, the stricter revolving-door policy. For intermediate values of $\lambda$, the principal may prefer to use $U r$ rather than $1 U$, as it involves a lower bonus. Figure 4 in the appendix graphically illustrates the principal's anti-capture policy as function of the parameters. It confirms the finding of the baseline model that a higher cost of raising public funds makes a stricter revolving-door policy more attractive, whereas a larger value of the regulatory expertise favors the adoption of a bonus policy. 


\subsection{Explicit Capture}

In this section, we suppose that the firm can attempt to influence the reporting strategy by using bribes or the promise of a post-agency job. Specifically, the firm can approach the expert before he sends his report to the principal with a take-it-or-leave-it explicit sidecontract: a bribe $b$ to report $r=\emptyset$. The cost of arranging and enforcing the side-contract, $(1-\tau) b$, is lost, where $\tau \in[0,1) \cdot{ }^{21}$

There are two main takeaways. ${ }^{22}$ First, the firm would either use the bribe or the revolving door to capture the experts but not both.

Lemma 1. There exists a threshold function

$$
\hat{\tau}(\delta):=\frac{\delta(1-q \eta)}{1-\delta q \eta}
$$

increasing in $\delta$, such that for $\tau$ above (below) the threshold the firm would only use the bribe (salary) to capture the expert.

And second, as a direct consequence:

Corollary 1. A better (yet inefficient) bribing technology may prevent the firm from using the revolving door to capture the experts.

The intuition is that the implicit promise of a post-agency salary is not credible if the firm will be able to resort to a slightly inefficient third-party enforcement mechanism in the future that enables explicit capture. Note that when either $\tau$ or $\delta$ are sufficiently high, the principal will refrain from preventing capture.

Importantly, policies other than the bonus scheme that can prevent capture, such as those which affect $\tau$, like whistleblowing protection policies or higher-quality monitoring, and $w_{M a x}$, like cooling-off periods should be designed bearing in mind how capture is enforced. The policies seem to be complementary: a substitution between the two means of capture may take place if the policy maker only changes one policy.

\footnotetext{
${ }^{21}$ While we treat this parameter as exogenous, in practice the ease with which the collusive partners can enforce the explicit side contract depends on institutional and cultural factors, like the scrutiny of the firm-expert interaction, external monitoring, whistleblowing protection policies, as well as penalties for the parties that are found guilty.

${ }^{22}$ Detailed derivations and discussions can be found in Section B.7 of the Appendix.
} 
The same intuition also provides an important implication for commissions and kickbacks: policies that promote capping or banning these (as suggested by e.g., Inderst and Ottaviani, 2012c) may be ineffective if a firm can simply hire or promote the advisors or salespeople whose recommendation it wants to influence.

If there is frequent interaction between a firm and a regulatory agency, the revolving door is the favorite (and possibly unique) channel used by the firm to capture experts. However, a more efficient bribing technology or an authority that does not monitor bribes leads to bribes being used rather than the revolving door. This scenario appears to best fit (large parts of) the developing world. Uncertainty about firms' survival prospects and scarce resources available to monitoring and implementing regulation (as argued by Estache and Wren-Lewis, 2009) contribute to making bribes so notoriously rife in less developed countries. By contrast, in most richer countries, thanks to superior monitoring and better enforcement of well-established policies, explicit capture is a less pressing issue. However, as firms typically have a longer life-span, the credibility of their promises to reward accommodating experts is reinforced, making implicit capture the chief concern. ${ }^{23}$

In Figure 3, we graphically represent the parameter regions where capture is tolerated or deterred and which kind of policies the principal uses to deter capture or which channel the firm uses. Note that we have defined

$$
\hat{\delta}:=\frac{(1-\eta)(D-G+\underline{c})+\lambda \underline{c}}{q(1-\eta) \eta(D-G+\underline{c})+q \lambda[(1-\eta) G+\eta \underline{c}]},
$$

and it is derived from $\hat{\lambda}$, whereas $\bar{\tau}$ is the positive root that we can obtain from $\tilde{\lambda}$ as characterized in Lemma 2 in Appendix B.7, i.e., the threshold of $\lambda$ above which the principal prefers to tolerate explicit capture rather than prevent it. ${ }^{24}$

\footnotetext{
${ }^{23}$ In this respect, there is a complementarity with the paper by Harstad and Svensson (2011). Harstad and Svensson (2011) develop a model that can explain why bribery is relatively more prevalent in poor countries and lobbying is more commonly found in rich ones, whereas our model can account for why capture is more likely to occur via bribes in poor countries and via the revolving door in rich ones.

${ }^{24}$ The figure is drawn from the following parameter values: $q=0.5, \eta=0.8, \underline{c}=0.5, G=20, D=30$, $\lambda=1, v=15, \bar{u}=0$.
} 


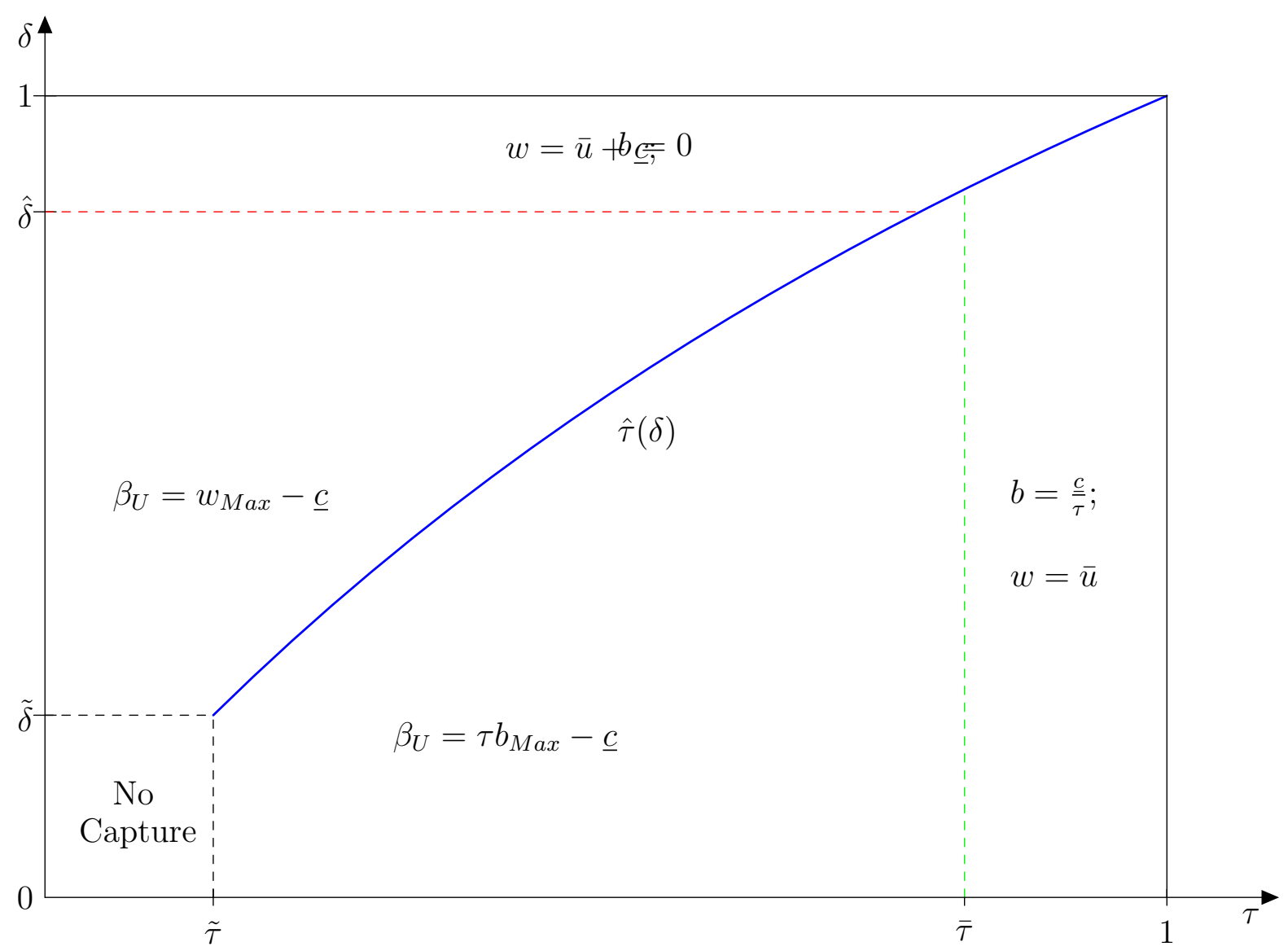

Figure 3: Equilibria and Interplay between $\tau$ and $\delta$.

\section{Concluding Remarks}

In this paper we have studied how firms can use the revolving-door channel to capture experts. So far, the informative role played by the revolving door has been overlooked. We have shown that this channel is more efficient than bribes to sway regulatory outcomes: recruiting former regulators can be a very powerful commitment device, signaling to current and future experts the firm's eagerness to reward a lenient behavior. It is also more efficient than bribes in that the revolving door can emerge as an industry norm that does not require an explicit and illegal agreement between the firm-experts coalition. However, the more efficient the explicit side-contract, the less likely it is that the firm can use superior implicit agreements.

We have highlighted that the desirability of keeping the revolving door open depends on the frequency of the firm-regulatory agency interaction. When this is sporadic, the revolving door should better be open. As capture would require an explicit agreement, 
standard anti-corruption policies, such as whistleblowing protection, could be used to deter collusion. If interaction is frequent, an implicit understanding between regulators and the regulated firm can sustain capture via the revolving door. The lack of smoking-gun evidence means that regulatory capture could be deterred either by generously rewarding experts who report information that is unfavorable to the firm or by closing the revolving door. The pros and cons of closing the revolving door should be weighed against each other. If the regulatory experts' contribution to the industry or their opportunity cost of working for the agency is high, it may be socially beneficial to tolerate capture. This is especially the case if the likelihood that the experts can actually misreport information is low. In this regard, we have shown that opening the door selectively, namely, conditionally on the information revealed by the experts can be welfare improving. It is straightforward but noteworthy that regulatory capture may also lead to a misallocation of human resources if the capturing firm hires experts who would be better placed in other firms or institutions. This would make capture deterrence even more valuable.

One might argue that firms may hire experts to get a more direct and efficient access to their (then former) colleagues (e.g., see Blanes i Vidal et al., 2012). According to this view, a policy preventing former experts from interacting with their previous colleagues, or working on cases directly related to their previous job, would make the firm less likely to hire experts, whereas in our model, this policy should not have any effect. If direct access to experts is problematic, such a policy could be complementary to selectively closing the revolving door. As employment restrictions reducing access are already in place in some agencies, it would be possible to test the relative importance of either channel empirically (signaling/monitoring versus access).

Another important question that we leave open for future research is how competition among firms affects the optimal policies to deal with regulatory capture. A firm might have an incentive to induce experts to truthfully report unfavorable evidence about other firms to shut them down. At the same time, the firms' incentives to collude with one another change, which in turn may make regulatory capture harder to deter. Thus, the effects of competition on regulatory capture are unclear and may depend on several institutional factors. However, we expect the mechanisms of our model to continue to play a role. As we implicitly model a competitive fringe via the expert's outside option 
$\bar{u}$, we conjecture that our results continue to hold at least in settings where the expertise an expert acquires at the regulatory agency is very firm-specific or the products firms sell are very differentiated.

In the model, we have assumed that the experts' reports are used to determine whether the firm can be authorized to produce. While this best fits the case of experts charged with assessing a firm's eligibility for patents or licenses, the framework can be adapted to analyze other contexts where regulatory capture is a concern, from public procurement to tax auditing, from financial supervision to firms' compliance with existing laws. Thirdparty certification is another suitable setting to which the findings of our model can be applied, as certifiers may distort information to benefit or harm sellers (e.g., see Dranove and Jin, 2010 and references therein). Our framework could even be reinterpreted to analyze the transition of politicians to the private sector - in this case, the citizenry would be the principal and the politicians would be the experts. Lastly, we believe that our findings also speak to the current debate about the anonymity of referee reports: allowing or requiring referees to disclose their identity may enable referees to publicly signal their willingness to engage in and stick to a mutually beneficial exchange of favors, which may increase biases in the refereeing process.

\section{A Appendix A}

\section{Proof of Proposition 1}

In the first-best solution, the principal sets $\beta_{U}=\beta_{\emptyset}=0$. Suppose first that the principal does not alter the bonus policy. The maximum salary that the firm can credibly promise to pay, $w_{\emptyset}^{M a x}$ is derived from making (1) bind. This must be at least as large as $\underline{c}$ to induce low-concealment cost experts to hide evidence. Therefore, if $\delta<\tilde{\delta}$ there is no room for capture - part (a) of the proposition.

To show part (b) of the proposition, suppose now that $\delta \geq \tilde{\delta}$ so that capture may occur. If the principal decides to tolerate capture, it is better not to reward experts because $\lambda>0$. It follows that the firm will offer the minimum wage that induces capture, i.e., $w=\underline{c}$ and $W^{\text {RDtolerate }}$ is obtained. Alternatively, the principal will prevent capture by setting $\Delta_{\beta}=\beta_{U}-\beta_{\emptyset}>w_{\emptyset}^{\text {Max }}-\underline{c}$. To minimize the cost of capture deterrence, $\beta_{\emptyset}=0$ and 
$W^{R d b o n u s}$ is obtained. The principal prefers to deter capture when $W^{R D b o n u s} \geq W^{R D t o l e r a t e}$, that is, when $\lambda \leq \hat{\lambda}$. The threshold $\hat{\lambda}$ is decreasing in $\delta$. To see this, note that

$$
\frac{\partial \hat{\lambda}}{\partial \delta}=\frac{-q(1-\eta)^{2} G(D-G+\underline{c})}{[\delta q(1-\eta) G-(1-\delta q \eta) \underline{c}]^{2}}<0 .
$$

\section{Proof of Proposition 2}

It directly follows from comparing welfare expressions.

\section{Proof of Proposition 3}

For a bribery implicit contract that induces experts to conceal evidence to be self-enforcing it must be that the following expert's capture incentive compatibility holds, that is, a low concealment cost expert must prefer to manipulate evidence that the state is unsafe:

$$
\mu_{b} b \geq \beta_{U}-\beta_{\emptyset}+\underline{c}
$$

where $\mu_{b}$ is the expert's belief that the firm will make good on the promise. The firm's capture-incentive compatibility constraint requires that:

$$
-(1-\delta) b+\delta \frac{1-\delta}{1-\delta \mu_{b}}\left[\mu_{b} \pi^{R D}+\left(1-\mu_{b}\right) \pi^{S G}\right] \geq \delta \frac{1-\delta}{1-\delta \mu_{b}}\left[\mu_{b} \pi^{R D}+\left(1-\mu_{b}\right) \pi^{S G}\right] .
$$

Paying the bribe does not affect future experts' belief that the firm makes good on the promises. Therefore, the only credible bribe is $b=0$, but this cannot induce experts to manipulate evidence if $\Delta_{\beta} \geq 0$ and $\underline{c} \geq 0$, with at least one strict inequality.

\section{Proof of Remark 1}

Suppose that at the end of each period, the period $t$ expert can send a message $m_{t} \in$ $\{C, N C\}$ that is observed by all future experts. An expert sends message $C$ if the firm has paid the implicitly agreed-upon bribe and $N C$, otherwise. A low-concealment cost expert who has observed $s=U$ conceals information only if $C$ has been reported by all previous experts. Then, it is possible to construct an equilibrium where capture is self-enforcing 
if $\delta$ is high enough. Suppose that $\Delta_{\beta}=0$ and $b=\underline{c} \geq 0$, if $r=\emptyset$, and no bribe is paid otherwise. Then, if

$$
\begin{aligned}
\delta\left(\pi^{R D}-\pi^{S G}\right) & \geq(1-\delta) \underline{c} \\
\Leftrightarrow G & \geq \frac{1-\delta q \eta}{\delta q(1-\eta)} \underline{c},
\end{aligned}
$$

then neither the firm nor the experts are willing to deviate. If a deviation has occurred, the experts will not conceal evidence anymore: they earn the same irrespective of what they do and can force the firm to its min-max payoff.

\section{Proof of Stationarity}

First note that the firm cannot influence experts with $c=\infty$. Now consider a situation in which the firm manages to let the low manipulation cost expert misreport the signal in period $t$ in the derived stationary equilibrium. Because the expert does not care about any future periods and is just indifferent between misreporting or not in $t$, there is no cheaper way for the firm to influence this expert. Moreover, the firm could not benefit from a different report and, because we look at an equilibrium, the cost for the firm does not exceed its benefit from misreporting. Now consider a situation in which the firm does not want to let the low manipulation cost expert misreport the signal in period $t$ in the derived stationary equilibrium. Trivially, the equilibrium wage is the cheapest wage the firm can offer. If the firm wanted to influence the experts, the cheapest way would be using the wage $w$ as in the analysis for the stationary equilibrium because the expert does not care about any future periods and would just be indifferent. However, by the optimality of the equilibrium, the cost for the firm exceeds its benefit from misreporting. Thus, there is no situation in which the firm could do better.

\section{B Appendix B}

\section{B.1 Proof of Remark 2}

The according conditions are unchanged. 


\section{B.2 Proof of Proposition 4}

The principal again has four different options.

Tolerating capture. In this case, the principal does not offer a bonus and welfare is:

$$
W_{U E \text { ffort }}^{\text {RDtorate }}=G-q D+v \text {. }
$$

Note that the principal does not induce effort and thus no expert acquires information. If the principal wanted to provide enough incentives without closing the revolving door at least selectively, this would be equivalent to the reward scheme case below (in which there is no capture).

Reward scheme. The principal may decide to prevent capture by setting a reward for $r=U$. This is achieved with the following bonus schedule: $\beta_{\emptyset \text { Effort }}^{\text {RGder }}=0$ and

$$
\beta_{U E f f o r t}^{R D \text { deter }}=\max \left\{\frac{c_{e}}{q}, \frac{\delta q(1-\eta) G}{1-\delta q \eta}-\underline{c}, 0\right\} \geq \beta_{U}^{R \text { Ddeter }}
$$

which may be more expensive than if high-ability experts detect the unsafe state automatically. Welfare is:

$$
W_{U E f f o r t}^{R D d e t e r}=(1-q) G+v-\lambda q \beta_{U E f f o r t}^{R D d e t e r}-c_{e} .
$$

Blanket ban. A blanket ban only makes sense if the principal also induces effort. This solution gives:

$$
W_{U E f f o r t}^{R D \text { blanketban }}=(1-q) G-\lambda q \beta_{U E f f o r t}^{R D b l a n k e t b a n}-c_{e}, \text { where }
$$

$\beta_{\emptyset \text { Effort }}^{\text {RDblanketban }}=0$ and $\beta_{U E \text { ffort }}^{R \text { Dblanketban }}=c_{e} / q$.

Conditional ban. Also a conditional ban only makes sense if effort induced.

$$
W_{U E f f o r t}^{R D \text { conditionalban }}=(1-q) G+q v-\lambda q \beta_{U E f \text { fort }}^{R D \text { conditionalban }}-c_{e},
$$

which is strictly higher than $W_{U E \text { ffort }}^{R D \text { blankan }}$, where $\beta_{\emptyset \text { ff fort }}^{R D \text { condionalban }}=0$ and $\beta_{U E f \text { fort }}^{R D \text { conditionalban }}=$ $\max \left\{c_{e} / q-\bar{u}, 0\right\}$. The principal does not have to pay such a high bonus if the inspector is also motivated by future wages. The principal prefers to (selectively) keep the revolving door open if:

$$
(1-q) v>\lambda q\left(\beta_{U E f f o r t} U^{R D d e t e r}-\beta_{U E f \text { fort }}^{\text {RDconaliban }}\right) .
$$


If $c_{e}$ is small, this is the same condition as in the case in which the high-ability expert automatically learns the unsafe state.

The statement of the proposition directly follows from comparing welfare expressions.

\section{B.3 Moral Hazard on safety care}

We assume that, in each period $t$, the firm privately decides whether or not to dedicate resources to improve product safety. If the firm does not invest, the good is unsafe with baseline probability $q_{0}$, whereas if the firm invests at cost $\phi>0$, the probability that the activity is unsafe reduces to $q_{1}<q_{0} \cdot{ }^{25}$ It holds that $1>1-q_{1}>1-q_{0}>0$ and we set $G>q_{0} D$. Hence, investing does not rule out the possibility that the good brings about damages and, if there were no information about the state of the world, the principal would allow production. To make the problem interesting, we also assume that

$$
G \in\left(\frac{\phi}{q_{0}-q_{1}}, \frac{\phi}{\left(q_{0}-q_{1}\right) \eta}\right)
$$

so that the firm would invest in the stage game, whereas it would not in the repeated game if it succeeded in capturing the experts. ${ }^{26}$ Thus, capture is associated with the twin evils of excessive authorization and under-investment in safety. The firm's expected profit if capture takes place is

$$
\pi_{q E n d}^{R D}=\left(1-q_{0} \eta\right)\left(G-w_{\emptyset}\right)-q_{0} \eta w_{U}+v
$$

and the maximum self-enforcing salary is derived from making the firm's dynamic captureincentive compatibility constraint bind:

$$
w_{\emptyset}^{M a x}=\bar{u}+\frac{\delta\left[\left(q_{1}-q_{0} \eta\right) G+\phi\right]}{1-\delta q_{0} \eta},
$$

since $w_{U}$ will continue to be set equal to $\bar{u}$. For capture to be an issue, it must be that $\delta>\tilde{\delta}_{q E n d}$, where

$$
\tilde{\delta}_{q E n d}:=\frac{\underline{c}}{\left(q_{1}-q_{0} \eta\right) G+\phi+q_{0} \eta \underline{c}} .
$$

\footnotetext{
${ }^{25}$ For simplicity, we assume that the firm's investment decision is binary. Having a continuous investment function would not qualitatively alter the main conclusions of the analysis.

${ }^{26}$ In fact, the condition is overly restrictive: since the firm needs to pay a premium to an expert who reports $\emptyset$, the right-bound of the interval can be higher.
} 
When it is an issue, tolerating capture would yield:

$$
W_{q E n d}^{R \text { Dolerate }}=\left(1-q_{0} \eta\right) G-q_{0}(1-\eta)(D+\underline{c})+v
$$

To prevent capture, the principal will contemplate two options. First, paying a bonus to an expert who reports $r=\emptyset$. Specifically, setting $\beta_{U}^{R D b o n u s}=0$ and $\beta_{\emptyset}^{R D b o n u s}=$ $\frac{\delta\left[\left(q_{1}-q_{0} \eta\right) G+\phi\right]}{1-\delta q_{0} \eta}-\underline{c}$ both deters capture and restores optimal investment incentives. Welfare is

$$
W_{q E n d}^{R D \text { bonus }}=\left(1-q_{1}\right) G-\lambda q_{1}\left(\frac{\delta\left[\left(q_{1}-q_{0} \eta\right) G+\phi\right]}{1-\delta q_{0} \eta}-\underline{c}\right)+v-\phi .
$$

Note that an increase in the investment cost makes resorting to the bonus policy less convenient, whereas the effect of an increase in the investment gain, $q_{0}-q_{1}$, unequivocally increases welfare under this solution.

The principal could also prevent capture by prohibiting experts who have made favorable reports from joining the industry at the end of their term at the agency. By adopting this policy, the principal need not use positive bonuses to reward the expert, i.e., $\beta_{\emptyset}=\beta_{U}=0$. However, with a conditional ban, the firm would invest in product safety only if:

$$
G \geq \frac{\phi}{q_{0}-q_{1}}+(v-\bar{u})
$$

Welfare is

$$
W_{q E n d}^{R D \text { conditionalban }}= \begin{cases}\left(1-q_{1}\right) G+q_{1} v-\phi, & \text { if } v-\bar{u}<G-\frac{\phi}{\left(q_{0}-q_{1}\right)} \\ \left(1-q_{0}\right) G+q_{0} v, & \text { otherwise. }\end{cases}
$$

Comparing welfare expressions: preventing capture with a bonus scheme is preferred to tolerating capture if

$$
\lambda \leq \hat{\lambda}_{q E n d} \equiv \frac{\left.\left(1-\delta q_{0} \eta\right)\left[q_{0}(1-\eta)(D+\underline{c})-\left(q_{1}-q_{0} \eta\right) G+\phi\right]\right]}{q_{1} \delta\left[\left(q_{1}-q_{0} \eta\right) G+\phi\right]-q_{1}\left(1-\delta q_{0} \eta\right) \underline{c}} .
$$

When $v-\bar{u}$ is below the threshold, selectively closing the revolving door is preferred to the bonus policy if

$$
v<\hat{v}_{q E n d 1} \equiv \lambda \frac{q_{1}}{1-q_{1}} \frac{\delta\left[\left(q_{1}-q_{0} \eta\right) G+\phi\right]-\left(1-q_{0} \delta \eta\right) \underline{c}}{\left(1-q_{0} \delta \eta\right)}
$$

The conditional ban dominates tolerating capture for:

$$
v \leq \tilde{v}_{q E n d 1} \equiv \frac{\left(q_{0} \eta-q_{1}\right) G+q_{0}(1-\eta)(D+\underline{c})-\phi}{1-q_{1}}
$$


which coincides with the previous threshold for $\lambda=\hat{\lambda}_{q E n d}$.

If $v-\bar{u}$ is high enough so that the conditional ban would lead to a low investment, the conditional ban outperforms tolerating capture if

$$
v<\tilde{v}_{q E n d 0} \equiv \frac{q_{0}}{1-q_{0}}(1-\eta)(D+\underline{c}-G)
$$

Moreover, it outperforms the bonus policy if:

$$
v<\hat{v}_{q E n d 0} \equiv \lambda \frac{q_{1}}{1-q_{0}}\left(\frac{\delta\left[\left(q_{1}-q_{0} \eta\right) G+\phi\right]}{1-\delta q_{0} \eta}-\underline{c}\right)-\frac{\left(q_{0}-q_{1}\right) G-\phi}{1-q_{0}} .
$$

Note that for $\lambda=\hat{\lambda}_{q E n d}$, the two thresholds $\hat{v}_{q E n d 0}$ and $\tilde{v}_{q E n d 0}$ coincide. We summarize the results of this extension in the following proposition.

Proposition 6. Suppose that $\delta \geq \tilde{\delta}_{q E n d}$ and $v-\bar{u}>(\operatorname{resp} . \leq) G-\frac{\phi}{\left(q_{0}-q_{1}\right)}$.

1. If $\lambda \leq \hat{\lambda}_{q E n d}$, the principal prevents regulatory capture by

(a) selectively closing the revolving door if $v<\hat{v}_{q E n d 0}$ (resp., $\left.v<\hat{v}_{q E n d 1}\right)$;

(b) using the bonus policy if $v \geq \hat{v}_{q E n d 0}$ (resp., $\left.v \geq \hat{v}_{q E n d 1}\right)$.

2. If $\lambda>\hat{\lambda}_{q \text { End }}$, the principal

(a) prevents capture by selectively closing the revolving door if $v \leq \tilde{v}_{q E n d 0}$ (resp., $\left.v<\tilde{v}_{q E n d 1}\right)$

(b) tolerates capture if $v>\tilde{v}_{q E n d 0}$ (resp., $\left.v>\tilde{v}_{q E n d 1}\right)$.

\section{B.4 Experts versus Bureaucrats - technical details}

Obviously, in the stage game, there is no welfare gain from hiring bureaucrats. To see this, note that welfare would drop to $W_{B}^{S G}=(1-q \epsilon) G-q(1-\epsilon) D+v_{B}$, where we use the subscript $B$ to refer to the employment of bureaucrats, rather than experts. In the repeated game, the emergence of capture may provide a rationale for hiring bureaucrats.

We characterize the principal's favorite policy when all officials are bureaucrats.

Proposition 7. Capture is an issue only for $\delta>\tilde{\delta}_{B}$ and

1. If $\lambda \leq \hat{\lambda}_{B}$, the principal prevents regulatory capture by 
(a) selectively closing the revolving door if $v_{B}<\hat{v}_{B}$;

(b) using the bonus policy if $v_{B} \geq \hat{v}_{B}$.

2. If $\lambda>\hat{\lambda}_{B}$, the principal

(a) prevents capture by selectively closing the revolving door if $v_{B} \leq \tilde{v}_{B}$;

(b) tolerates capture if $v_{B}>\tilde{v}_{B}$.

Proof. Suppose that only bureaucrats are available. The firm's capture-incentive dynamic constraint is:

$$
(1-\delta)\left(v_{B}-w_{\emptyset}^{B}\right)+\delta \pi_{B}^{R D} \geq(1-\delta)\left(v_{B}-\bar{u}_{B}\right)+\delta \pi_{B}^{S G}
$$

where

$$
\pi_{B}^{R D}=(1-q \epsilon \eta)\left(G-w_{\emptyset}^{B}\right)-q \epsilon \eta w_{U}^{B}+v_{B},
$$

and $\pi_{B}^{S G}=(1-q \epsilon) G+v_{B}-\bar{u}_{B}$. It follows that the maximum self-enforcing salary $w_{\emptyset}^{B-M a x}$ that the firm could credibly promise to pay is:

$$
w_{\emptyset}^{B-M a x}:=\bar{u}_{B}+\frac{\delta q \epsilon(1-\eta) G}{1-\delta q \epsilon \eta} .
$$

The salary must also be such that the bureaucrat is willing to conceal unfavorable evidence:

$$
w_{\emptyset}^{B}-\bar{c}+\beta_{\emptyset}^{B}>w_{U}^{B}
$$

With bureaucrats, capture is an issue for $\delta>\tilde{\delta}_{B}:=\frac{\underline{c}}{q \epsilon(1-\eta) G+q \epsilon \eta \underline{c}}$. When an issue, the principal can tolerate capture getting

$$
W_{B}^{R D \text { tolerate }}=(1-q \epsilon \eta) G-q(1-\epsilon \eta) D-q \epsilon(1-\eta) \underline{c}+v_{B}
$$

The principal could decide to deter capture by using the bonus policy or by conditionally banning bureaucrats that have provided favorable information from taking up a position in the industry. In the latter case, welfare would be:

$$
W_{B}^{R D \text { conditionalban }}=(1-q \epsilon) G+q \epsilon v_{B}-q(1-\epsilon) D .
$$

Lastly, a bonus policy consisting of $\beta_{\emptyset}^{B}=0$ and $\beta_{U}^{B}=\frac{\delta q \epsilon(1-\eta) G}{1-\delta q \epsilon \eta}-\underline{c}$ prevents capture and yields:

$$
W_{B}^{R D b o n u s}=(1-q \epsilon) G-q(1-\epsilon) D-\lambda q \epsilon\left(\frac{\delta q \epsilon(1-\eta) G}{1-\delta q \epsilon \eta}-\underline{c}\right)+v_{B}
$$


By comparing welfare expressions, it is easy to check that the bonus policy is preferred to tolerating capture when

$$
\lambda \leq \hat{\lambda}_{B}:=\frac{(1-\eta)(1-\delta q \epsilon \eta)(D-G+\underline{c})}{\delta q \epsilon(1-\eta) G-(1-\delta q \epsilon \eta) \underline{c}} .
$$

Moreover, $W_{B}^{R D \text { conditionalban }}>W_{B}^{R D b o n u s}$ for:

$$
v<\hat{v}_{B}:=\lambda \frac{q \epsilon}{1-q \epsilon}\left(\frac{\delta q \epsilon(1-\eta) G}{1-\delta q \epsilon \eta}-\underline{c}\right)
$$

and $W_{B}^{R \text { Dconditionalban }} \geq W_{B}^{R \text { Dtolerate }}$ for:

$$
v \leq \tilde{v}_{B}:=\frac{q \epsilon}{1-q \epsilon}(1-\eta)(D-G+\underline{c})
$$

It also easy to see that $\tilde{v}_{B}=\hat{\lambda}_{B} \hat{v}_{B}$.

We now determine when the principal may benefit from hiring bureaucrats rather than experts.

Remark 7. The principal may prefer to hire bureaucrats to either (i) avoid capture being an issue or (ii) reduce the cost of the bonus policy.

Proof. (i) For $\delta \in\left(\tilde{\delta}, \tilde{\delta}_{B}\right]$, capture is an issue only if the principal employs experts to monitor the firm. See that: $W_{B}^{S G}>W^{R D t o l e r a t e}$ if $q(\epsilon-\eta)(D-G)>\left(v-v_{B}\right)-q(1-\eta) \underline{c}$; $W_{B}^{S G}>W^{R D \text { conditionalban }}$ if $v_{B}-q v>q(1-\epsilon)(D-G)$; and $W_{B}^{S G}>W^{R D b o n u s}$ if

$$
\lambda q\left(\frac{\delta q(1-\eta) G}{1-\delta q \eta}-\underline{c}\right)>q(1-\epsilon)(D-G)+\left(v-v_{B}\right) .
$$

All inequalities are more likely to hold when $\epsilon$ is higher and $v_{B}$ is closer to $v$, that is the inefficiency of the bureaucrats is not excessive.

(ii) See that $W_{B}^{R D b o n u s}$ is not necessarily increasing in $\epsilon$, because it may increase the total bonus bill. Let $\epsilon^{\text {RDbonus }}$ be the level of $\epsilon \in[0,1]$ that maximizes $W_{B}^{R \text { Dbonus }}$. Note that $\epsilon^{\text {RDbonus }}$ is implicitly determined from the first-order condition:

$$
q(D-G)-\lambda q\left(\frac{\delta q \epsilon(1-\eta) G}{1-\delta q \epsilon \eta}-\underline{c}\right)-\lambda q \epsilon \frac{\delta q(1-\eta) G}{(1-\delta q \epsilon \eta)^{2}}=0
$$

The second-order condition is negative and it is possible to show that $\frac{\partial \epsilon^{R D b o n u s}}{\partial D}>0$, $\frac{\partial \epsilon^{R D b o n u s}}{\partial G}<0, \frac{\partial \epsilon^{R D b o n u s}}{\partial \underline{c}}>0$, whereas $\frac{\partial \epsilon^{R D b o n u s}}{\partial \lambda}<0$ if $G$ is relatively higher than $\underline{c}$. As $\epsilon^{R D b o n u s}$ may be less than one, the principal may prefer to hire bureaucrats instead of 
experts. It is also straightforward to see that $W_{B}^{R D c o n d i t i o n a l b a n}<W^{R D \text { conditionalban }}$. Lastly, note that

$$
W^{R D \text { tolerate }} \geq W_{B}^{R \text { Dtolerate }} \Leftrightarrow D-G \geq \frac{(1-\eta)}{\eta} \underline{c}-\frac{v-v_{B}}{q \eta(1-\epsilon)} .
$$

Note that $D-G<\frac{(1-\eta)}{\eta} \underline{c}$ would imply that $\frac{\partial W_{B}^{R D t o l e r a t e}}{\partial \epsilon}<0$, so that the principal would be better off by hiring clueless officials, that is, by shutting down the agency.

\section{B.5 Cooling-off periods}

Post-employment restrictions. Title 18 of the U.S. Code in Section 207 establishes general post-employment restrictions on former officers, employees, and elected officials of the executive agencies of the United States. These include (i) a permanent ban on influencing any agency's or court's decision in connection with a particular matter in which the person participated personally; (ii) a two-year ban concerning those matters that were pending under the person's responsibility up to year after leaving office; (iii) a one-year ban on senior officials to influence the department or agency in which the person worked for any matters for which the person seeks official action. Procurement officers are subject to further restrictions: they cannot accept compensation from the contractor of a procurement of $\$ 10,000,000$ or more within one year of working on the procurement. There may also be different restrictions for specific regulators. For instance, Tenekedjieva (2019) documents quite some variation in the legal restrictions U.S. State commissioners (some of whom are appointed by the State governors) face when they leave office, such as temporary ban on lobbying or on assisting formerly regulated firms.

In the European Union (EU), the legislation is essentially decentralized to the single state members. This has resulted in a great deal of heterogeneity, as emphasized by a Transparency International report that focuses on lobbying (Mulcahy, 2015). The report assesses the regulation of the lobbying practice in the EU. Among the metrics taken into account, there is integrity, namely, the rules that govern the ethical conduct of lobbyists and public officials. The results are rather grim. First, lobbyists and public officials are not subject to clear and enforceable rules regarding their activity: some countries do not even have a code of conduct for public sector employees. Many countries have adopted cooling-off periods for some public officials. For instance, in France, the law requires a 
three-year cooling-off period before a public official could join a firm that he or she was previously responsible for in terms of surveillance or control activities. Only legislators in many states in the US are subject to cooling-off periods, whereas in Europe only Slovenia has rolled out a cooling-off period for MPs. Lastly, the report also finds that the provisions are generally poorly enforced.

\section{Proof of Remark 3}

Take the derivative of $W_{\text {cool }}^{R D \text { deter }}$ with respect to $\gamma$ is:

$$
\frac{\partial W_{\text {cool }}^{R D d e t e r}}{\partial \gamma}=-\frac{\left(1-q \mathbb{1}_{\text {select }}\right) v}{(1+\gamma)^{2}}-\lambda q \frac{\partial \beta_{U}^{R D d e t e r}(\gamma)}{\partial \gamma}
$$

It follows that $\gamma$ would optimally set equal to zero if $\frac{\partial \beta_{U}^{R D d e t e r}(\gamma)}{\partial \gamma} \geq 0$.

\section{B.5.1 Proof of Proposition 5}

We start by determining welfare under unconditional cooling-off periods. We need to find the maximum salary the firm would be willing to pay to an expert who is accommodating. If there is regulatory capture:

$$
\pi_{c o o l}^{R D}=(1-q \eta)\left(G-\frac{w_{\emptyset}}{1+\gamma}\right)+\frac{v-q \eta \bar{u}}{1+\gamma}
$$

If regulatory capture cannot be sustained $\pi_{\text {cool }}^{S G}=(1-q) G+\frac{(v-\bar{u})}{1+\gamma}$. Therefore,

$$
\delta\left(\pi_{\text {cool }}^{R D}-\pi_{\text {cool }}^{S G}\right)=\delta q(1-\eta) G-\delta(1-q \eta) \frac{w_{\emptyset}-\bar{u}}{1+\gamma} .
$$

The maximum salary the firm would be willing to pay is

$$
w_{\emptyset}^{M a x}=\bar{u}+\frac{(1+\gamma) \delta q(1-\eta) G}{1-\delta q \eta+\gamma(1-\delta)} .
$$

To prevent capture, the principal could set $\beta_{\emptyset}^{R D \text { deter }}=0$ and $\beta_{U}^{R D d e t e r}=\max \left\{\frac{w_{\emptyset}-\bar{u}}{1+\gamma}-\underline{c}, 0\right\}$. As a result,

$$
\beta_{U}^{R D d e t e r}(\gamma)=\max \left\{\frac{\delta q(1-\eta) G}{1-\delta q \eta+\gamma(1-\delta)}-\underline{c}, 0\right\}
$$

We now determine welfare under report-contingent cooling-off periods. To distinguish this solution, in the rest of the proof, we use the subscript select. Profit under regulatory capture is:

$$
\pi_{\text {select }}^{R D}=(1-q \eta)\left(G+\frac{v-w_{\emptyset}}{1+\gamma}\right)+q \eta(v-\bar{u})
$$


If regulatory capture cannot be sustained:

$$
\pi_{\text {select }}^{S G}=(1-q) G+\frac{(1-q)(v-\bar{u})}{1+\gamma}+q(v-\bar{u}) .
$$

Therefore,

$$
\pi_{\text {select }}^{R D}-\pi_{\text {select }}^{S G}=q(1-\eta) G-\frac{(1-q \eta)\left(w_{\emptyset}+\gamma v\right)}{1+\gamma}+\frac{[1+q \gamma-q \eta(1+\gamma)] \bar{u}}{1+\gamma} .
$$

The maximum salary the firm would be willing to pay is

$$
w_{\emptyset}^{M a x}=\frac{(1+\gamma) \delta q(1-\eta) G-\delta q \gamma(1-\eta) v-[\delta \gamma(1-q)-(1-\delta q \eta)(1+\gamma)] \bar{u}]}{\delta(1-q \eta)+(1+\gamma)(1-\delta)} .
$$

To prevent capture, the principal could set $\beta_{\emptyset}^{R D S e l e c t}=0$ and $\beta_{U}^{R D S e l e c t}=\max \left\{\frac{w_{\emptyset}}{1+\gamma}-\bar{u}-\underline{c}, 0\right\}$. The effect of an increase in $\gamma$ on $\beta_{U}^{R D \text { Select }}$ is:

$$
\frac{\partial \beta_{U}^{R D S e l e c t}}{\partial \gamma}=\frac{1}{1+\gamma} \frac{\partial w_{\emptyset}^{\text {Max }}}{\partial \gamma}-\frac{w_{\emptyset}^{\text {Max }}}{(1+\gamma)^{2}}
$$

The second term is negative, reflecting how a longer cooling-off period blunts the influence of future industry payments on the expert's report. The expert discounts the lure of receiving a higher salary from the regulated firm compared to what he could receive from other employers in the industry. However, a longer cooling-off period tends to increase the maximum salary the firm would be willing to pay, that is, the first term:

$$
\frac{\partial w_{\emptyset}^{M a x}}{\partial \gamma}=\frac{q \delta(1-\eta)[\delta(1-q \eta) G-(1-\delta q \eta)(v-\bar{u})]}{[1+\gamma(1-\delta)-q \delta \eta]^{2}}
$$

This is because the longer cooling-off period actually increases the gap between the continuation payoffs, $\pi_{\text {select }}^{R D}$ and $\pi_{\text {select }}^{S G}$, by deferring the payment of the salary to the accommodating expert. As a result, a longer cooling-off period can make the firm more willing to keep its promises. As the overall effect tends to be negative, the principal may want to opt for a positive $\gamma$.

To prove that the cooling-off period should be made conditional on the expert's report, see that $^{27}$

$$
\begin{aligned}
& W_{\text {select }}^{\text {RDdeter }}>W_{\text {cool }}^{R D \text { deter }} \\
\Leftrightarrow & \frac{\gamma v}{1+\gamma}>\lambda q\left[\beta_{U}^{R D \text { select }}-\beta_{U}^{R D d e t e r}\right] \\
& \frac{\gamma v}{1+\gamma}>-\lambda q\left(\frac{\gamma \delta(1-\eta) v+\gamma[1-q \delta+\gamma(1-\delta)] \bar{u}}{(1+\gamma)[1+\gamma(1-\delta)-q \delta \eta]}\right) .
\end{aligned}
$$

\footnotetext{
${ }^{27}$ We make the comparison for positive bonuses, for otherwise it is not optimal to set a positive coolingoff period, regardless of its conditionality on the report.
} 
Thus, in what follows we restrict attention to report-contingent cooling-off periods. If $\beta_{U}^{R D \text { Select }}>0$, consider the first order condition of the principal's problem for an interior optimum:

$$
Z^{\text {select }}(\gamma) \equiv-\frac{(1-q) v}{(1+\gamma)^{2}}-\lambda q \frac{\partial \beta_{U}}{\partial \gamma}=0
$$

The second order condition is negative for $\lambda$ sufficiently high. We now use the implicit function theorem to study how changes in the primitives affect $\gamma$ at the interior optimum. As the second-order condition must be satisfied, the sign of $\frac{\partial \gamma^{U}}{\partial \lambda}$ will coincide with the sign of $\frac{\partial Z^{\text {select }}}{\partial \lambda}$ :

$$
\frac{\partial Z^{\text {select }}}{\partial \lambda}=-q \frac{\partial \beta_{U}}{\partial \gamma}>0
$$

as $\beta_{U}$ must be decreasing in $\gamma$ at the interior solution. Consider now the impact of an increase in $G$ on the equilibrium value of $\gamma$ :

$$
\frac{\partial Z^{\text {select }}}{\partial G}=-\lambda q \frac{\partial^{2} \beta_{U}}{\partial \gamma \partial G}>0
$$

because $\frac{\partial^{2} \beta_{U}}{\partial \gamma \partial G}<0$. To see this note that:

$$
\begin{aligned}
\frac{\partial^{2} \beta_{U}^{R D S e l e c t}}{\partial \gamma \partial G} & =\frac{1}{1+\gamma} \frac{\partial^{2} w_{\emptyset}^{\text {Max }}}{\partial \gamma \partial G}-\frac{1}{(1+\gamma)^{2}} \frac{\partial w_{\emptyset}^{\text {Max }}}{\partial G} \\
& =\frac{q \delta^{2}(1-\eta)(1-q \eta)}{(1+\gamma)[1+\gamma(1-\delta)-q \delta \eta]^{2}}-\frac{q \delta(1+\gamma)(1-\eta)}{(1+\gamma)^{2}[1+\gamma(1-\delta)-q \delta \eta]} \\
& =-\frac{q \delta(1-\delta)(1-\eta)}{[1+\gamma(1-\delta)-q \delta \eta]^{2}}<0 .
\end{aligned}
$$

An increase in $v$ has an ambiguous impact on the equilibrium value of $\gamma$ :

$$
\begin{aligned}
\frac{\partial Z^{\text {select }}}{\partial v} & =-\frac{(1-q)}{(1+\gamma)^{2}}-\lambda q\left[\frac{1}{1+\gamma} \frac{\partial^{2} w_{\emptyset}^{\text {Max }}}{\partial \gamma \partial v}-\frac{1}{(1+\gamma)^{2}} \frac{\partial w_{\emptyset}^{\text {Max }}}{\partial v}\right] \\
& =-\frac{(1-q)}{(1+\gamma)^{2}}-\lambda q\left[-\frac{q \delta(1-\eta)(1-q \delta \eta)}{(1+\gamma)[1+\gamma(1-\delta)-q \delta \eta]^{2}}+\frac{q \delta \gamma(1-\eta)}{(1+\gamma)^{2}[1+\gamma(1-\delta)-q \delta \eta]}\right] \\
& =-\frac{(1-q)}{(1+\gamma)^{2}}+\lambda q \frac{q \delta(1-\eta)\left[1-\gamma^{2}(1-\delta)-q \delta \eta\right]}{(1+\gamma)^{2}[1+\gamma(1-\delta)-q \delta \eta]^{2}} .
\end{aligned}
$$

It can also be shown that an increase in $\bar{u}$ has an ambiguous effect on the equilibrium bonus and, consequently, on the equilibrium value of $\gamma$.

\section{B.6 Multiple per-period reports}

Consider the firm's payoff in the stage game, where there is no capture: $\pi^{S G 2}=2(1-$ q) $G+v-\bar{u}$ and social welfare is $W^{F B 2}=2(1-q) G+v$, where we have added 2 to the superscript denoting the solution, as there are two signals. 
The tightest firm's dynamic capture-incentive compatibility constraint occurs when an expert has reported $r^{1}=r^{2}=\emptyset$, since it plausibly holds that $w_{\emptyset \emptyset} \geq \max \left\{w_{\emptyset U}, w_{U \emptyset}\right\} \geq$ $\min \left\{w_{\emptyset U}, w_{U \emptyset}\right\} \geq w_{U U}:$

$$
(1-\delta)\left(v-w_{\emptyset \emptyset}\right)+\delta \pi^{R D 2} \geq(1-\delta)(v-\bar{u})+\delta \pi^{S G 2}
$$

where $\pi^{R D 2}$ denotes the firm's expected profit from adhering to the revolving-door implicit contract is:

$$
\pi^{R D 2}=2(1-q \eta) G-(1-q \eta)^{2} w_{\emptyset \emptyset}-(1-q \eta) q \eta\left(w_{\emptyset U}+w_{U \emptyset}\right)-q^{2} \eta^{2} w_{U U}+v
$$

Therefore,

$\Delta_{\pi 2}:=\pi^{R D 2}-\pi^{S G 2}=2 q(1-\eta) G-(1-q \eta)^{2} w_{\emptyset \emptyset}-(1-q \eta) q \eta\left(w_{\emptyset U}+w_{U \emptyset}\right)-q^{2} \eta^{2} w_{U U}+\bar{u}$.

Consider now the expert's capture incentive compatibility constraints. The expert must be willing to conceal an unfavorable signal whenever the cost of concealment is low. Thus, irrespective of his first report, the expert must be willing to incur $\underline{c}$ to conceal unfavorable evidence in Stage 3

$$
w_{r \emptyset}-\underline{c}+\beta_{r \emptyset}>w_{r U}+\beta_{r U}
$$

for $r \in\{U, \emptyset\}$. That is,

$$
w_{\emptyset \emptyset}-\underline{c}+\beta_{\emptyset \emptyset}>w_{\emptyset U}+\beta_{\emptyset U}
$$

when $r^{1}=\emptyset$, and

$$
w_{U \emptyset}-\underline{c}+\beta_{U \emptyset}>w_{U U}+\beta_{U U}
$$

when $r^{1}=U$. The expert must also be willing to conceal evidence for his first report, without knowing with certainty the second signal he will collect as well as its manipulability:

$$
(1-q \eta)\left(w_{\emptyset \emptyset}+\beta_{\emptyset \emptyset}\right)+q \eta\left(w_{\emptyset U}+\beta_{\emptyset U}\right)-c>(1-q \eta)\left(w_{U \emptyset}+\beta_{U \emptyset}\right)+q \eta\left(w_{U U}+\beta_{U U}\right) .
$$

Let us first determine when regulatory capture is an issue that inevitably decreases welfare. Namely, suppose that the revolving door is open and the principal does not offer a positive bonus. The firm would set the minimum salaries that satisfy (B2), (B3), and (B4). It is immediate to see that the salary schedule which make all these constraints bind is: 
$w_{\emptyset \emptyset}=2 c+\bar{u}, w_{U \emptyset}=w_{\emptyset U}=c+\bar{u}, w_{U U}=\bar{u}^{28}$ Replacing these wages in (B1), we find that deterring capture is costless if $\delta \leq \tilde{\delta}$.

If $\delta>\tilde{\delta}$, but the principal does not take any measure to prevent regulatory capture, welfare is:

$$
W^{R D 2 t o l e r a t e}=2(1-q \eta) G-2 q(1-\eta)(D+\underline{c})+v .
$$

Because of Inequality (9), this is always dominated by some other options. We now show that $U r$ dominates $r U$.

\section{Proof of Remark 5}

Consider first option $U r$. For partial capture to be an equilibrium, it must be that the expert is willing to conceal unfavorable evidence in stage 2.b:

$$
w_{U \emptyset}+\beta_{U \emptyset}-\underline{c}>w_{U U}+\beta_{U U},
$$

and that the firm is willing to honor the promise of paying a higher salary to an expert whose second report has been favorable:

$$
(1-\delta)\left(v-w_{U \emptyset}\right)+\delta \pi_{U r}^{R D 2} \geq(1-\delta)(v-\bar{u})+\delta \pi_{U r}^{S G 2}
$$

Bear in mind that with this revolving-door policy, if there is no capture the firm obtains $\pi_{U r}^{S G 2}=2(1-q) G+q(v-\bar{u})$, because the expert can be hired only if $r^{1}=U$, which occurs with probability $q$. If the firm succeeds in partially capturing the experts, it expects to get:

$$
\pi_{U r}^{R D 2}=(1-q) G+q(1-q \eta) G+(1-q)^{2} G-q(1-q \eta) w_{U \emptyset}-q^{2} \eta w_{U U}+q v
$$

Therefore, the highest salary the firm would be willing to pay is:

$$
w_{U \emptyset}^{M a x}=\bar{u}+\frac{\delta q^{2}(1-\eta) G}{1-\delta+\delta q(1-q \eta)} .
$$

Partial capture is an issue only if $\delta>\frac{c}{q^{2}(1-\eta) G+[1-q(1-q \eta)] \underline{c}}$. In that case, to prevent capture in Stage 3 through the bonus policy, it is easy to see that the principal should set $\beta_{U U}=$ $\frac{\delta q^{2}(1-\eta) G}{1-\delta+\delta q(1-q \eta)}-\underline{c}$ and all the other bonuses equal to 0 . Welfare would be:

$$
W_{U r}^{R D 2 b o n u s}=2(1-q) G-\lambda q^{2}\left[\frac{\delta q^{2}(1-\eta) G}{1-\delta+\delta q(1-q \eta)}-\underline{c}\right]+q v .
$$

\footnotetext{
${ }^{28}$ In fact, to induce capture the firm will have to pay the expert slightly more than those salaries.
} 
With $r U$, for partial capture to be an equilibrium, it must be that the expert is willing to conceal unfavorable evidence in stage 2.a, knowing that there will never be concealment in stage 2.b:

$$
q\left(w_{\emptyset U}+\beta_{\emptyset U}\right)+(1-q) \beta_{\emptyset \emptyset}-\underline{c}>q\left(w_{U U}+\beta_{U U}\right)+(1-q) \beta_{U \emptyset}
$$

Moreover, the firm must find it profitable to make good on the promised salary if $r^{1}=\emptyset$ and $r^{2}=U$ :

$$
(1-\delta)\left(v-w_{\emptyset U}\right)+\delta \pi_{r U}^{R D 2} \geq(1-\delta)(v-\bar{u})+\delta \pi_{r U}^{S G 2}
$$

Note that, with this revolving-door policy, if there is no capture the firm obtains $\pi_{r U}^{S G 2}=$ $2(1-q) G+q(v-\bar{u})$. If partial capture occurs, the firm expects to obtain:

$$
\pi_{r U}^{R D 2}=(1-q \eta) G+(1-q) G-(1-q \eta) q w_{\emptyset U}-q^{2} \eta w_{U U}+q v
$$

Therefore, the highest salary the firm would be willing to pay is:

$$
w_{\emptyset U}^{M a x}=\bar{u}+\frac{\delta q(1-\eta) G}{1-\delta+\delta q(1-q \eta)},
$$

which is higher than $w_{U \emptyset}^{M a x}$. We retrieve exactly the same threshold as with the other policy $\delta>\frac{c}{q^{2}(1-\eta) G+[1-q(1-q \eta)] \underline{c}}$. If the above inequality holds and the principal prevents partial capture with a bonus, she'd better set $\beta_{\emptyset U}=\beta_{\emptyset \emptyset}=0$ and can set $\beta_{U U}=\beta_{U \emptyset}=$ $\frac{\delta q(1-\eta) G}{1-\delta+\delta q(1-q \eta)}-\underline{c}^{29}$ Welfare is:

$$
W_{r U}^{R D 2 b o n u s}=2(1-q) G-\lambda q\left[\frac{\delta q^{2}(1-\eta) G}{1-\delta+\delta q(1-q \eta)}-\underline{c}\right]+q v .
$$

The expected wage bill is always higher than with $U r$. Therefore, the principal would never adopt $r U$ because it makes partial-capture prevention more expensive.

We now illustrate the two remaining options.

Option $1 U$. Firstly, consider that the firm cannot fully capture an expert, since he cannot be rewarded if $r^{1}=r^{2}=U$. Partial capture could be an equilibrium, though. The following constraints need to be satisfied if the firm wants to influence the expert as often as possible: first, the expert must be willing to conceal unfavorable evidence in stage 2.b, if $r^{1}=U$ :

$$
w_{U \emptyset}+\beta_{U \emptyset}-\underline{c}>w_{U U}+\beta_{U U} ;
$$

\footnotetext{
${ }^{29}$ The principal can also pay any combination of $\beta_{U U}$ and $\beta_{U \emptyset}$ such that $q \beta_{U U}+(1-q) \beta_{U \emptyset}=$ $\frac{\delta q^{2}(1-\eta) G}{1-\delta+\delta q(1-q \eta)}-\underline{c}$ to prevent partial capture with the same welfare effects.
} 
and the expert must be willing to conceal unfavorable evidence in stage 2.a:

$$
q\left(w_{\emptyset U}+\beta_{\emptyset U}\right)+(1-q) \beta_{\emptyset \emptyset}-\underline{c}>(1-q \eta)\left(w_{U \emptyset}+\beta_{U \emptyset}-\underline{c}\right)+q \eta\left(w_{U U}+\beta_{U U}\right) .
$$

The firm must be willing to honor the promise of paying a higher salary to an expert whose report has been favorable:

$$
(1-\delta)\left(v-w_{U \emptyset}\right)+\delta \pi_{1 U}^{R D 2} \geq(1-\delta)(v-\bar{u})+\delta \pi_{1 U}^{S G 2}
$$

and

$$
(1-\delta)\left(v-w_{\emptyset U}\right)+\delta \pi_{1 U}^{R D 2} \geq(1-\delta)(v-\bar{u})+\delta \pi_{1 U}^{S G 2}
$$

Note that

$$
\pi_{1 U}^{S G 2}=2(1-q) G+q(2-q)(v-\bar{u})
$$

and

$$
\pi_{1 U}^{R D 2}=(1-q \eta) G+(1-q \eta)(1-q) G+q \eta(1-q \eta) G-(1-q \eta) q w_{\emptyset U}-q^{2} \eta^{2} w_{U U}-q \eta(1-q \eta) w_{U \emptyset}+q[1+\eta(1-q)] v
$$

We can now compute the surplus that partial capture may bring about:

$\pi_{1 U}^{R D}-\pi_{1 U}^{S G}=q(1-\eta)(1+q \eta) G-(1-q \eta) q w_{\emptyset U}-q \eta(1-q \eta) w_{U \emptyset}-q(1-q)(1-\eta) v+q\left[2-q\left(1+\eta^{2}\right)\right] \bar{u}$

To determine when capture is an issue, set $\beta_{r r}=0$ for any $r^{1}, r^{2}$. Note that $w_{U \emptyset}=\bar{u}+c$ to induce evidence concealment in stage 2.b. Then, to conceal evidence in stage 2.a, $w_{\emptyset U} \geq \frac{\bar{u}+c}{q}$. Replacing $w_{U \emptyset}=\bar{u}+c$ in the second dynamic-enforcement constraint, we can recover $w_{\emptyset U}^{M a x}$ and we find that corruption is an issue only if

$$
\delta>\frac{c+(1-q) \bar{u}}{q^{2}(1-\eta)(1+q \eta) G+\left(1-q+q^{3} \eta^{2}\right) c-q^{2}(1-q)(1-\eta) v+(1-q)[1-(1-q) q] \bar{u}} .
$$

It is possible to show that, plugging $w_{U \emptyset}=\bar{u}+c$ and $w_{\emptyset U}=\frac{\bar{u}+c}{q}$ in the first dynamicenforcement constraint, this holds for a lower level of $\delta$.

Moreover, policy $1 U$ opens up further capture opportunities for the firm. First, the firm may decide to promise a reward only conditional on a favorable report in stage 2.b, thereby relinquishing to induce concealment of an unfavorable signal in stage 2.a. In that case, to convince the expert to conceal evidence:

$$
w_{U \emptyset}+\beta_{U \emptyset}-\underline{c}>w_{U U}+\beta_{U U} .
$$


While this looks like an option we studied earlier, the firm has a strengthened incentive to keep its promise because the revolving-door policy is more advantageous:

$\pi_{1 U}^{R D 2 o n l y 2}=(1-q) G+q(1-q \eta) G+(1-q)^{2} G-q(1-q \eta) w_{U \emptyset}-(1-q) q w_{\emptyset U}-q^{2} \eta w_{U U}+\left[1-(1-q)^{2}\right] v$.

Alternatively, the firm may promise to reward an expert for a favorable report in stage 2 . If so,

$$
q\left(w_{\emptyset U}+\beta_{\emptyset U}\right)+(1-q) \beta_{\emptyset \emptyset}-\underline{c}>q\left(w_{U U}+\beta_{U U}\right)+(1-q)\left(w_{U \emptyset}+\beta_{U \emptyset}\right) .
$$

By capturing the expert for the first report,

$\pi_{1 U}^{R D 2 o n l y 1}=(1-q \eta) G+(1-q) G-(1-q \eta) q w_{\emptyset U}-q^{2} \eta w_{U U}-q \eta(1-q) w_{U \emptyset}+[1-(1-q \eta)(1-q)] v$.

Let us now determine the bonus policy to prevent capture under $1 U$. It is beneficial to set $\beta_{\emptyset \emptyset}=0$. Note that with this revolving-door policy, the principal incentivizes one unfavorable report sufficiently. Therefore, she optimally only sets a positive $\beta_{U U}$ and sets all other bonuses equal to zero. In particular, the principal does not gain from setting $\beta_{\emptyset U}>0$ : as the expert can join the firm only if at least one report is unfavorable, he will never conceal evidence in stage $2 . \mathrm{b}$, if $r_{1}=\emptyset$. Setting $\beta_{U \emptyset}>0$ helps prevent concealment in stage 2.a but makes stage-2.b concealment more attractive. Thus, the principal should set $\beta_{\emptyset \emptyset}=\beta_{\emptyset U}=\beta_{U \emptyset}=0$ and $\beta_{U U}>0$. In particular, $\beta_{U U}$ must be so high that the firm cannot capture the expert either in stage 2.a or stage 2.b. To this end,

$$
\beta_{U U} \geq \max \left\{w_{\emptyset U}^{M a x}-\frac{\bar{u}+\underline{c}}{q}, w_{U \emptyset}^{M a x}-\underline{c}-\bar{u}\right\} .
$$

We now determine these maximum salaries the firm could promise to pay. The maximum $w_{U \emptyset}$ is determined from the firm's dynamic capture-incentive compatibility constraint when capture is induced in stage 2.b only:

$$
-(1-\delta)\left(w_{U \emptyset}-\bar{u}\right)+\delta\left(\pi_{1 U}^{R D 2 o n l y 2}-\pi_{1 U}^{S G 2}\right) \geq 0 .
$$

Since

$$
\pi_{1 U}^{R D 2 o n l y 2}-\pi_{1 U}^{S G 2}=q^{2}(1-\eta) G-q(1-q \eta)\left(w_{U \emptyset}-\bar{u}\right)
$$

the maximum salary that satisfies the constraint is:

$$
w_{U \emptyset}^{M a x}=\bar{u}+\frac{\delta q^{2}(1-\eta) G}{1-\delta+\delta q(1-q \eta)},
$$


and, as a result, to prevent capture in stage 3 :

$$
\beta_{U U} \geq \frac{\delta q^{2}(1-\eta) G}{1-\delta+\delta q(1-q \eta)}-\underline{c}
$$

The maximum $w_{\emptyset U}$ is determined from the firm's dynamic capture-incentive compatibility constraint when capture is induced in stage 2.a only:

$$
-(1-\delta)\left(w_{\emptyset U}-\bar{u}\right)+\delta\left(\pi_{1 U}^{R D 2 o n l y 1}-\pi_{1 U}^{S G 2}\right) \geq 0
$$

Since

$$
\pi_{1 U}^{R D 2 o n l y 1}-\pi_{1 U}^{S G 2}=q(1-\eta) G-(1-q \eta) q w_{\emptyset U}-q(1-q)(1-\eta) v+q(2-q-\eta) \bar{u}
$$

the maximum salary that satisfies the constraint is:

$$
w_{\emptyset U}^{M a x}=\frac{\delta q(1-\eta)[G-(1-q) v]}{1-\delta+\delta q(1-q \eta)}+\frac{1-\delta+\delta q(2-q-\eta)}{1-\delta+\delta q(1-q \eta)} \bar{u} .
$$

To prevent capture in stage 2.a:

$$
\beta_{U U} \geq w_{\emptyset U}^{M a x}-\frac{\underline{c}+\bar{u}}{q}
$$

Preventing capture in stage 2.a is more costly and, accordingly, the bonus must be set to avert that type of capture when $G$ is sufficiently large as compared to $\bar{u}, v$ and $\underline{c}$. However, for our scope, it is convenient to find a threshold expressed in terms of $v$, where a higher value of regulatory expertise only decreases the cost of preventing evidence concealment in stage 2.a whereas it does not affect the cost of deterring concealment in stage 2.b. Intuitively, by inducing evidence concealment in stage 2.a, the firm may not be able to hire the expert after all - this occurs if stage 2.b signal turns out to be . Thus, this option is less appealing to the firm when the value of regulatory expertise is higher. Specifically, define

$$
\tilde{v}(G, \underline{c}, \bar{u}) \equiv \frac{\delta^{2} q(1-\eta) G+[1-\delta+\delta q(1-q \eta)] \underline{c}-[1-\delta+(1-q) \delta q] \bar{u}}{q^{2} \delta(1-\eta)}
$$

Then, to prevent capture under this revolving-door policy, the principal must set:

$$
\beta_{U U}^{1 U \text { Bonus }}= \begin{cases}\frac{\delta q(1-\eta)[G-(1-q) v]}{1-\delta+\delta q(1-q \eta)}+\frac{1-\delta+\delta q(2-q-\eta)}{1-\delta+\delta q(1-q \eta)} \bar{u}-\frac{\underline{c}+\bar{u}}{q} & \text { if } v \leq \tilde{v}(G, \underline{c}, \bar{u}) \\ \frac{\delta q^{2}(1-\eta) G}{1-\delta+\delta q(1-q \eta)}-\underline{c} & \text { if } v>\tilde{v}(G, \underline{c}, \bar{u}) .\end{cases}
$$


By preventing capture, the principal obtains:

$$
W_{1 U}^{R D 2 \text { Bonus }}=2(1-q) G-\lambda q^{2} \beta_{U U}^{1 U \text { Bonus }}+\left[1-(1-q)^{2}\right] v
$$

It is immediate to see that $W_{1 U}^{R D 2 B o n u s}$ is greater than $W_{U r}^{R D 2 B o n u s}$ if $v>\tilde{v}(G, \underline{c}, \bar{u})$. Intuitively, the same bonus is needed to avert regulatory capture, whereas the benefit of revolving door, i.e., the valuable contribution of the expert to firm's profits is enjoyed more often when the revolving door policy does not establish beforehand in which period the expert's report must be unfavorable. If $v \leq \tilde{v}(G, \underline{c}, \bar{u})$ a trade-off arises in that policy $1 U$ entails a higher bonus. For this reason, $1 U$ is preferred only if $\lambda$ is sufficiently low. In particular, when $v \leq \tilde{v}(G, \underline{c}, \bar{u}), 1 U$ is preferred to $U r$ if and only if:

$$
\lambda<\frac{[1-\delta+\delta q(1-q \eta)] v}{\delta q^{2}(1-\eta)(G-v)-[1-\delta+\delta q(1-q \eta)] \underline{c}-[1-\delta+\delta q(1-q)] \bar{u}} .
$$

Option Bonus2. Lastly, suppose that the revolving door is open and the principal fully relies on bonuses to deter capture for both decisions. If so, the principal would choose the bonus schedule to maximize

$$
2(1-q) G-\lambda\left[(1-q)^{2} \beta_{\emptyset \emptyset}+q(1-q)\left(\beta_{U \emptyset}+\beta_{\emptyset U}\right)+q^{2} \beta_{U U}\right]+v
$$

subject to the following constraints:

$$
(1-q)\left(w_{U \emptyset}+\beta_{U \emptyset}\right)+q\left(w_{U U}+\beta_{U U}\right) \geq(1-q)\left(w_{\emptyset \emptyset}+\beta_{\emptyset \emptyset}\right)+q\left(w_{\emptyset U}+\beta_{\emptyset U}\right)-\underline{c},
$$

so that the expert will not find it advantageous to conceal unfavorable evidence in stage 2.a;

$$
w_{\emptyset U}+\beta_{\emptyset U} \geq w_{\emptyset \emptyset}+\beta_{\emptyset \emptyset}-\underline{c}
$$

so that the expert will not find it advantageous to conceal unfavorable evidence in stage 2.b, if $r^{1}=\emptyset$ and

$$
w_{U U}+\beta_{U U} \geq w_{U \emptyset}+\beta_{U \emptyset}-\underline{c}
$$

that ensure that an expert is unwilling to conceal unfavorable evidence in stage 2.b, if $r^{1}=U$. It is immediate to see that $\beta_{\emptyset \emptyset}=0$. Now, we need to determine the other salaries. From (B6),

$$
\beta_{\emptyset U} \geq w_{\emptyset \emptyset}-w_{\emptyset U}-\underline{c} .
$$


To determine $\beta_{\emptyset U}$, we need to find the most tempting salary schedule the firm could credibly promise to offer to the expert if it wants to induce concealment of unfavorable evidence in stage 2.b, following $r^{1}=\emptyset$. This is because the other constraints take care of deterring other capture opportunities. As $\pi^{S G 2}=2(1-q) G+v-\bar{u}$ and the profit associated with this type of capture is

$$
\pi_{\emptyset \emptyset \emptyset n l y 2}^{R D 2}=(1-q) G+(1-q)(1-q \eta) G+q(1-q) G+v-\bar{u}-(1-q)(1-q \eta)\left(w_{\emptyset \emptyset}-\bar{u}\right)
$$

it follows the profit gain is simply $(1-q) q(1-\eta) G-(1-q)(1-q \eta)\left(w_{\emptyset \emptyset}-\bar{u}\right)$ and, as a result,

$$
w_{\emptyset \emptyset}^{M a x}=\frac{\delta(1-q) q(1-\eta) G}{1-\delta+\delta(1-q)(1-q \eta)}+\bar{u}
$$

and

$$
\beta_{\emptyset U} \geq \frac{\delta(1-q) q(1-\eta) G}{1-\delta+\delta(1-q)(1-q \eta)}-\underline{c} .
$$

To determine $\beta_{U U}$ and $\beta_{U \emptyset}$ we first need to determine the most tempting salary schedule the firm could credibly promise to offer to the expert if it wants to induce concealment of unfavorable evidence in stage 2.b, following $r^{1}=U$, and then if the firm wants to induce concealment of unfavorable evidence in stage 2.a. The profit associated with capture occurring only in stage 2 .b if $r^{1}=U$ is:

$$
\pi_{\emptyset o n l y 2}^{R D 2}=(1-q) G+q(1-q \eta) G+(1-q)^{2} G+v-\bar{u}-q(1-q \eta)\left(w_{U \emptyset}-\bar{u}\right) .
$$

The profit gain is simply $q^{2}(1-\eta) G-q(1-q \eta)\left(w_{U \emptyset}-\bar{u}\right)$ and, as a result,

$$
w_{U \emptyset}^{M a x}=\frac{\delta q^{2}(1-\eta) G}{1-\delta+\delta q(1-q \eta)}+\bar{u} .
$$

Consider now the profit associated with capture occurring in stage 2 only:

$$
\pi_{U o n l y 1}^{R D 2}=(1-q \eta) G+(1-q) G+v-(1-q \eta) w_{\emptyset r}-q \eta \bar{u}
$$

and the profit gain is: $q(1-\eta) G-(1-q \eta)\left(w_{\emptyset_{r}}-\bar{u}\right)$. The maximum salary the firm could credibly promise to offer to induce concealment of the first report is:

$$
w_{\emptyset r}^{M a x}=\frac{\delta q(1-\eta) G}{1-\delta+\delta(1-q \eta)}+\bar{u}
$$

To determine $\beta_{U \emptyset}$ and $\beta_{U U}$ the following system of inequalities must be satisfied: from (B7)

$$
\beta_{U U}-\beta_{U \emptyset} \geq \frac{\delta q^{2}(1-\eta) G}{1-\delta+\delta q(1-q \eta)}-\underline{c}
$$


from (B5)

$$
q \beta_{U U}+(1-q) \beta_{U \emptyset} \geq \frac{\delta q(1-\eta) G}{1-\delta+\delta(1-q \eta)}-\underline{c}+q \beta_{\emptyset U} .
$$

A solution involves setting $\beta_{U \emptyset}=0$ and

$$
\beta_{U U}=\beta_{\emptyset U}+\frac{\delta(1-\eta) G}{\delta(1-\eta)-\delta+1}-\frac{c}{q} .
$$

In this way, (B5) binds whereas (B7) is slack. ${ }^{30}$ Welfare is:

$$
\begin{aligned}
W^{\text {Bonus } 2} & =2(1-q) G-\lambda\left[q(1-q) \beta_{\emptyset U}+q^{2} \beta_{U U}\right]+v \\
& =2(1-q) G-\lambda q\left(\frac{(1-\eta)\left[2-q(1+\delta)-\left(1-q^{2}\right) \eta \delta\right] q \delta G}{(1-\eta \delta)[1-q \delta-q \eta \delta(1-q)]}-2 \underline{c}\right)+v .
\end{aligned}
$$

\section{Proof of Remark 6}

The remark follows immediately from comparing the welfare expressions for options $U U$, Ur, $1 U$, and Bonus2.

Figure 4 graphically illustrates the principal's preferred anti-capture policy as function of $v$ and $\lambda{ }^{31}$ When $\lambda$ is relatively high and the value of the expert's regulatory expertise is low, the strictest revolving-door policy under which an expert can join the industry only if both $r^{1}=r^{2}=U$ dominates. When $\lambda$ is small, it is better to keep the revolving door open and rely on bonuses. For intermediate values of $\lambda$ and $v$, the principal prefers to use the policy $1 U$, where both bonuses and the closure of the revolving door are used to provide the experts with incentives to truthfully report unfavorable evidence.

\section{B.7 Explicit Capture}

In Subsection 3.2 we have shown that the firm cannot generally use bribes to capture experts in an implicit contract. This is because the firm will not face any punishment if it reneges on the promised bribe. However, bribes may be used if the firm and the experts have access to a third-party enforcement mechanism. This would require an explicit (not necessarily formal) contract that binds the collusive parties to what they

\footnotetext{
${ }^{30}$ It can be shown that setting $\beta_{U \emptyset}>0$ does not benefit the principal as it leads to a higher $\beta_{U U}$.

${ }^{31}$ In drawing the figure, we assume that $D=30, G=20, \underline{c}=0.5, \eta=0.8, q=0.5$, and $\delta=0.9$. Note that for the parameter values considered in the figure, solution $1 U$ always dominates $U r$.
} 


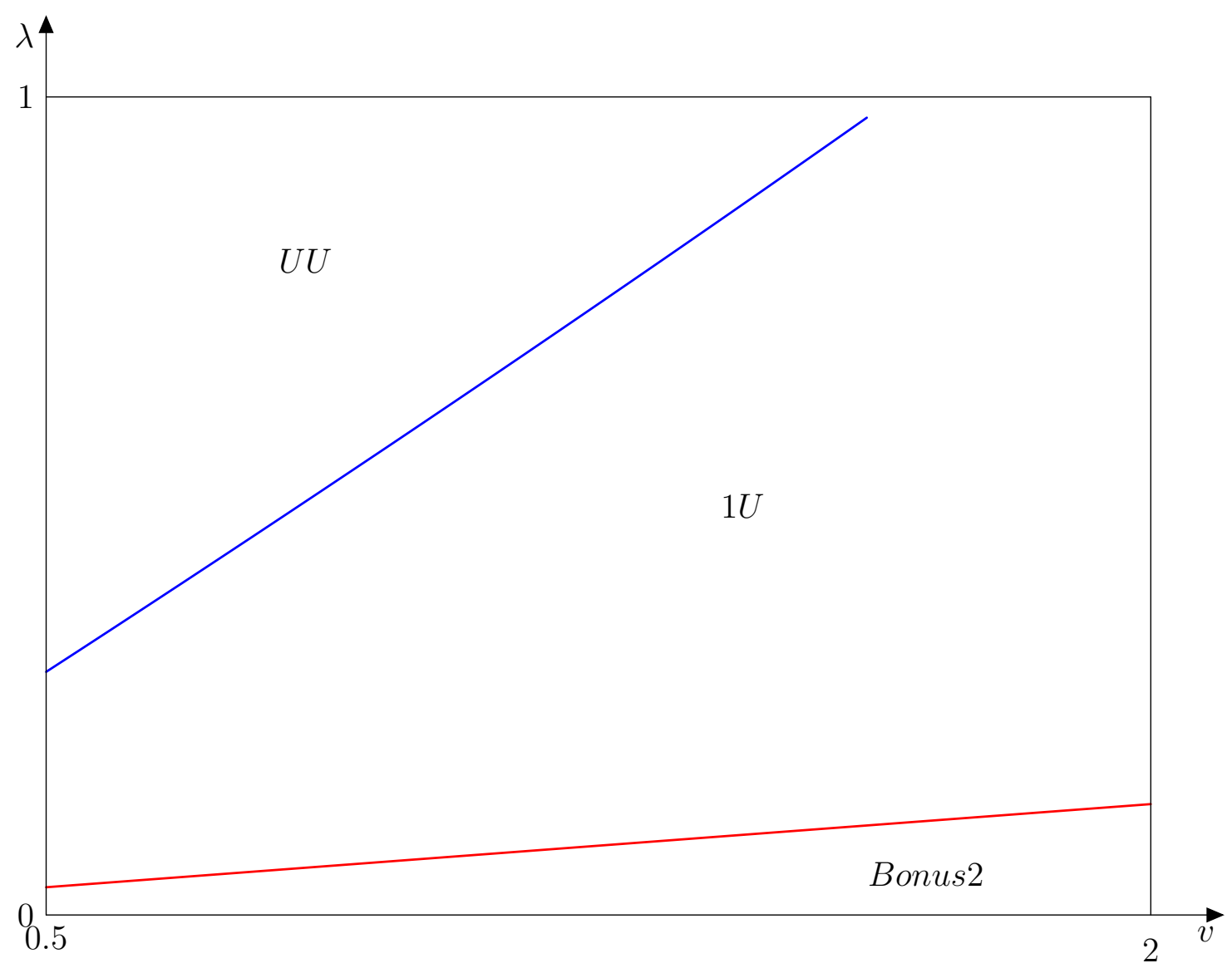

Figure 4: Anti-capture policies when each expert sends multiple reports.

have agreed upon. Indeed, most of the existing literature on corruption in hierarchies has relied on the assumption of some exogenous enforcement mechanism (e.g., Tirole, 1986). We now follow this literature by assuming the existence of some imperfectly enforceable mechanism the firm could resort to in order to capture the experts. This opens up the possibility of swaying the expert also in the absence of sufficiently frequent repeated interaction. However, the availability of this channel may thwart the use of superior implicit agreements in the repeated game.

This section discusses Subsection 4.7 more thoroughly, including the case in which the firm cannot use the revolving door but only bribes.

Note that in either case, the firm cannot effectively punish an expert by not hiring him as he would find employment in the industry and earn $u$. At the same time, the expert would not punish expert by refusing a job offer with a wage marginally larger than $u$. Thus, in equilibrium, the firm will always employ all experts because $v \geq u$. 


\section{B.7.1 One-stage Game}

Consider the firm's incentives to capture the expert via the side-contract in the absence of repeated interaction. If the firm does not bribe the expert and he reports truthfully the observed information, the firm's expected profit is $(1-q) G+v-u$. Conversely, if the firm's bribe $b$ leads the expert to hide unfavorable evidence when the concealment cost is low, the firm's expected profit is $(1-q \eta)(G-b)+v-u$. This is because the expert would report $r=\emptyset$ and pocket the firm's bribe when either $s=\emptyset$ or $s=U$ and $c=\underline{c}$. It follows that the maximum bribe the firm would be willing to pay is such that the firm is indifferent between offering the bribe and not engaging in the side contract:

$$
b_{M a x}=\frac{q(1-\eta)}{1-q \eta} G
$$

This bribe is only worth $\tau b_{\text {Max }}$ to the expert because of the transaction cost associated with organizing the side-contract.

The principal will then set the payment policy to either tolerate or fight corruption. Suppose the principal decides to prevent capture. If so, in order to induce the expert's participation, the following individual rationality constraint must be satisfied:

$$
q \beta_{U}+(1-q) \beta_{\emptyset}+u \geq 0
$$

Preventing capture requires that the expert prefers to report evidence that production is unsafe when evidence is manipulable, that is:

$$
\beta_{U} \geq \tau b+\beta_{\emptyset}-\underline{c}
$$

There is room for a profitable side-contract only if $\tau b_{\max } \geq \underline{c}$. Given that $b_{\max }=\frac{q(1-\eta)}{1-q \eta} G$, preventing corruption is an issue (because it inevitably reduces welfare) only if:

$$
\tau \geq \tilde{\tau} \equiv \frac{(1-q \eta) \underline{c}}{q(1-\eta) G}
$$

That is, the side-contract cannot be overly inefficient. Suppose that $\tau \geq \tilde{\tau}$. If the principal wants to deter collusion at the minimum cost, she should set $\beta_{U}=\tau \frac{q(1-\eta) G}{1-q \eta}-\underline{c}$ and $\beta_{\emptyset}=0$ and welfare would be:

$$
W^{S G d e t e r}=(1-q) G+v-u-\lambda q\left(\tau \frac{q(1-\eta) G}{1-q \eta}-\underline{c}\right) .
$$


To understand the above expression, note that $(1-q) G+v-u$ is the firm's expected profit; the expert expects to get utility $u=q \beta_{U}>0$ as the incentive constraint binds. In itself, this is merely a transfer and does not appear in the welfare expression. The last term only refers to the social cost of raising distortionary taxes to pay the expert.

If the principal tolerates capture, there is no need to pay the experts, that is, $\beta_{U}=\beta_{\emptyset}=$ 0. Capture always takes place with the minimum bribe that induces the low-manipulation cost expert to conceal evidence as the firm holds all the bargaining power. The bribe is $b=\frac{c}{\tau}$. Welfare is:

$$
W^{S G t o l e r a t e}=\eta(1-q) G+v-u+(1-\eta)[G-q D-q \underline{c}]-(1-q \eta) \frac{1-\tau}{\tau} \underline{c} .
$$

Under this option, capture is prevented only when $c=\infty$. With a bribe equal to $\underline{c} / \tau$, the firm's profit is $(1-q \eta)(G-\underline{c} / \tau)+v-u$. An expert expects to get $(1-\eta)\left[\beta_{\emptyset}-q \underline{c}+\tau b\right]+$ $\eta\left[q \beta_{U}+(1-q)\left(\beta_{\emptyset}+\tau b\right)\right]+u=(1-q) \underline{c}+u$. The manipulation cost $\underline{c}$ is incurred only with probability $(1-\eta) q$. However, bribery occurs with probability $(1-\eta q)$ bringing about a welfare loss due to the transaction costs of the side-contract. Lemma 2 describes the principal's optimal behavior.

Lemma 2. (a) If $\tau<\tilde{\tau}$, capture is not an issue and the principal induces the expert's participation and truthful reporting by offering $\beta_{U}=\beta_{\emptyset}=0$;

(b) If $\tau \geq \tilde{\tau}$, capture is an issue: the principal deters capture by setting $\beta_{U}=\tau \frac{q(1-\eta) G}{1-q \eta}-\underline{c}$ and $\beta_{\emptyset}=0$ if and only if:

$$
\lambda \leq \tilde{\lambda} \equiv \frac{(1-\eta) q(D-G)+\underline{c}\left[(1-\eta) q+(1-\eta q) \frac{1-\tau}{\tau}\right]}{q\left[\tau \frac{q(1-\eta) G}{1-q \eta}-\underline{c}\right]}
$$

where $\tilde{\lambda}$ is decreasing in $\tau$. Otherwise, the principal does not prevent capture and $\beta_{U}=\beta_{\emptyset}=0$.

Therefore, the higher $\tau$, the more likely it is that capture is costly to prevent. A higher $\tau$ reduces welfare attainable when capture is prevented and may make the side-contract even too costly to prevent. If so, that is, if the side-contract is tolerated, a further increase in $\tau$ is welfare increasing, because it means that the welfare loss due to the occurrence of collusion decreases. An increase in $\lambda$ makes it more likely that the principal foregoes to prevent the side contract. The threshold $\tilde{\lambda}$ is decreasing in $\tau$. Intuitively, a higher 
$\tau$ increases the cost of preventing capture and simultaneously reduces the welfare loss suffered when the side-contract takes place. This also means that the principal might provide incentives for the expert for intermediate values of $\tau$, but not for high and low values of $\tau$.

\section{B.7.2 Interplay between Self-enforcing and Imperfectly Enforceable Capture}

We now examine the repeated game, where the firm need not rely on an imperfectly enforceable side-agreement to capture the experts, but it can make use of the incentives that repeated interaction entails. In particular, the firm can exploit the public information provided by the report made by the expert and the ensuing firm's salary offer. The firm can make use of two distinct channels to sway the regulatory outcome: an imperfectly enforceable side-agreement (or, bribery) and implicit capture by paying higher post-agency salaries. In this subsection, we explore the interplay between the two channels.

We extend the definition of the revolving-door implicit contract, denoted by $R C$ and starting at date $t$, as follows. It specifies that: (i) experts with a low concealment cost, $c=\underline{c}$, always conceal unfavorable evidence; (ii) the firm rewards experts who report $r=\emptyset$ with a bribe and/or improved post-agency job prospects; (iii) if at period $t+l$ the expert reported $r=\emptyset$ and the firm did not improve his job prospects, then at $t+l^{\prime}$ with $l^{\prime} \geq l$, the experts always reveal unfavorable evidence unless they receive a sufficiently high bribe. The revolving-door implicit contract is self-enforcing if these strategies form a Perfect Bayesian Equilibrium of the continuation game.

Consider the different constraints that must be satisfied in order for capture to be an equilibrium of the repeated game. First, the expert's capture-incentive compatibility constraint determines the condition under which an expert that has collected evidence unfavorable to the firm and has a low concealment cost is willing to report $r=\emptyset$ :

$$
\tau b+w+\beta_{\emptyset}-\underline{c} \geq \beta_{U},
$$

that is, the offered bribe and the expected wage must at least compensate for the differential bonus the expert will receive from the principal, $\Delta_{\beta}=\beta_{U}-\beta_{\emptyset}$, as well as the concealment cost.

The expert must expect a non-negative utility from accepting the job at the agency:

$$
q \eta\left(\beta_{U}+u\right)+(1-q \eta)\left(\tau b+w+\beta_{\emptyset}\right)-q(1-\eta) \underline{c} \geq 0 .
$$


As for the firm's willingness to engage in capture, we have the following firm's capture participation constraint:

$$
q(1-\eta) G \geq(1-q \eta)(w-u+b)
$$

Lastly, there is the firm's capture incentive compatibility constraint:

$$
-(1-\delta)(w-u)+\delta \pi^{R C} \geq \delta \pi^{D}
$$

where

$$
\pi^{R C}=(1-q \eta)(G-(w-u)-b)+v-u
$$

whereas $\pi^{D}$ depends on $\tau$ and $\lambda$ :

$$
\pi^{D}= \begin{cases}(1-q) G+v-u, & \text { if } \tau<\tilde{\tau} \\ (1-q) G+v-u, & \text { if } \tau \geq \tilde{\tau} \text { and } \lambda \leq \tilde{\lambda} \\ (1-q \eta)\left(G-\frac{c}{\tau}\right)+v-u, & \text { if } \tau \geq \tilde{\tau} \text { and } \lambda>\tilde{\lambda}\end{cases}
$$

First, consider the case in which $\tau<\tilde{\tau}$ : this means that $\pi^{D}=(1-q) G$. From (B13) it follows that the maximum wage that the firm can credibly promise to pay is

$$
\tilde{w}_{M a x}=\frac{\delta[q(1-\eta) G-(1-q \eta) b]}{1-\delta q \eta}+u .
$$

Note how paying a higher bribe reduces the maximum salary the firm can credibly promise to pay. By comparing $\pi^{R C}$ and $\pi^{D}$, this implies that the firm wants to pay a bribe of at most

$$
\tilde{b}_{M a x}=\frac{q(1-\eta) G}{1-q \eta} .
$$

Thus, deterring capture comes at no cost if $\delta<\tilde{\delta}$. If both the efficiency of the bribing technology and the firm's patience are low, capture is not an issue. Otherwise, the cheapest way to deter capture is setting

$$
\beta_{U}=\frac{\delta q(1-\eta) G}{1-\delta q \eta}-\underline{c}
$$

The principal then optimally deters capture if and only if

$$
\lambda \leq \hat{\lambda} \text { if } \delta \geq \tilde{\delta}
$$

If the principal does not prevent capture, $w=\underline{c}+u$ and $b=0$ as in the case without any bribing technology. An inefficient bribing technology does not help the firm because 
increasing the bribe further tightens (B13) for $\delta<\tilde{\delta}$; and for $\delta \geq \tilde{\delta}$, the firm finds it cheaper to use the wage.

Next, note that the case in which $\delta<\tilde{\delta}$ and $\tau \geq \tilde{\tau}$ is analogous to the case in which the principal cannot effectively use the revolving door and Lemma 2 applies. The intuition is that, for $\tau \geq \tilde{\tau}$, the firm uses the bribe rather than the wage because the firm lacks commitment off the equilibrium path. This also implies that, if the principal does not deter capture, $w=u$ and $b=\frac{c}{\tau}$.

Consider now the case in which $\tau \geq \tilde{\tau}$ and $\lambda \leq \tilde{\lambda}$. Therefore, off-the-equilibrium path the principal would deter collusion. Let us see whether the firm can leverage the revolving door to improve on the profit of the equilibrium game. If the principal tolerates capture in the repeated game, she sets $\beta_{U}=\beta_{\emptyset}=0$ and the firm will choose $b$ and $w$ to satisfy (B10): $\tau b+w-u=\underline{c}$. If $\delta \geq \tilde{\delta}$, then $w=\underline{c}$ and $b=0$, as that salary can credibly be promised to the experts. Bear in mind that the firm prefers to use the salary instead of the bribe to influence the experts' reports, as there is no difference between what the firm pays and what the expert receives. Therefore, if (B13) is slack, the firm will only use $w$ to capture the experts and set $b=0$ : if $b$ were positive, the firm could reduce $b$ and increase $w$ by a smaller amount and continue to satisfy the constraint while increasing its profits. Suppose that the principal wants to prevent capture. If so, $\beta_{\emptyset}=0$ and $\beta_{U}$ must be set in such a way as to make capture not individually rational or incentive compatible for the coalition firm-expert. In this regard, it is useful to determine the value of the maximum payment that the firm can offer to the expert. In Lemma 1, we identify a threshold function $\hat{\tau}(\delta)$ such that the maximum payment be given only in form of a wage (respectively, a bribe) when $\tau$ is below (above) that cutoff. Recall that Lemma 1 states that there exists a threshold function

$$
\hat{\tau}(\delta):=\frac{\delta(1-q \eta)}{1-\delta q \eta}
$$

increasing in $\delta$, such that for $\tau$ above (below) the threshold the firm would only use the bribe (salary) to capture the expert. Therefore, if $\tau>\hat{\tau}(\delta)$, the maximum payment that the firm can offer to the expert is $b_{\text {Max }}$. Given that $\lambda<\tilde{\lambda}$, the principal would deter capture by setting $\beta_{U}=\tau b_{M a x}-\underline{c}$. By contrast, if $\tau<\hat{\tau}(\delta)$, the maximum payment that the firm can offer to the expert is $w_{\text {Max }}$ and the principal would deter capture by setting $\beta_{U}=w_{M a x}-u-\underline{c}$ if $\lambda \leq \hat{\lambda}$ and would tolerate capture otherwise. 
Suppose now that $\tau>\tilde{\tau}$ and $\lambda>\tilde{\lambda}$. Then $\pi^{D}=(1-q \eta)(G-\underline{c} / \tau)+v-u$. Intuitively, the firm could always resort to the imperfectly enforceable side-contract to capture the experts. Note that this represents the firm's fall-back position as there is a stream of experts. Therefore, if the firm deviates from the proposed equilibrium strategies, there will always be the possibility to capture future experts with bribes. As the experts are distinct players, they cannot coordinate their punishments and commit to move to the worst equilibrium outcome (i.e., the one in which there is no side-contract) following the firm's deviation in one period. As a consequence, the firm cannot obtain less than $(1-q \eta)(G-\underline{c} / \tau)+v-u$ in each period following a deviation. The profit differential is

$$
\Delta_{\pi}=(1-q \eta)\left[\frac{\underline{c}}{\tau}-(w-u+b)\right]
$$

and the (B13) is written as:

$$
w-u \leq \frac{\delta(1-q \eta)(\underline{c} / \tau-b)}{1-\delta q \eta}
$$

If the principal does not prevent capture, the firm would set $w=\underline{c}+u$ and $b=0$ to induce the experts to conceal evidence. Only $w$ will be used if (B13) is satisfied:

$$
\tau \leq \frac{\delta(1-q \eta)}{1-\delta q \eta}=\hat{\tau}(\delta)
$$

Therefore, for

$$
\tau \in\left[\frac{(1-q \eta) \underline{c}}{q(1-\eta) G}, \frac{\delta(1-q \eta)}{1-\delta q \eta}\right],
$$

setting $w=\underline{c}+u$ and $b=0$ suffices to induce implicit capture. Note that the upper bound of the interval is greater than $\tilde{\delta}$, so this interval may exist. For $\tau$ above the threshold, the firm will only use the bribe.

\section{Proof of Lemma 2}

Part a) - suppose that the principal sets $\beta_{U}=\beta_{\emptyset}=0$. The maximum bribe that the firm is willing to offer, $b_{\text {Max }}$, is such that the firm is indifferent between capturing or not the experts. This must be at least as large as $\underline{c}$ to induce low-concealment cost experts to hide evidence. Therefore, if $\tau b_{\text {Max }}<\underline{c}$, capture never takes place. This inequality holds as long as $\tau<\tilde{\tau}$.

Part b) Suppose now that $\tau \geq \tilde{\tau}$ so that capture may occur. If the principal decides to tolerate capture, it is better not to reward experts because $\lambda>0$. It follows that the firm 
will offer the minimum bribe that induces capture, i.e., $b=\frac{c}{\tau}$ and $W^{\text {SGtolerate }}$ is obtained. Alternatively, the principal will prevent capture by setting $\Delta_{\beta}=\beta_{U}-\beta_{\emptyset}>\tau b_{M a x}-\underline{c}$. To minimize the cost of capture deterrence, $\beta_{\emptyset}=0$ and $W^{S G d e t e r}$ is obtained. The principal prefers to deter capture when $W^{\text {SGdeter }} \geq W^{\text {SGtolerate }}$, that is, when $\lambda \leq \tilde{\lambda}$. The threshold $\tilde{\lambda}$ is decreasing in $\tau$. To see this, note that:

$$
\frac{\partial \tilde{\lambda}}{\partial \tau}=\frac{q\left(\tau \frac{q(1-\eta)}{1-q \eta} G-\underline{c}\right) \underline{c}(1-\eta q)\left[\frac{-1}{\tau^{2}}\right]-\frac{q^{2}(1-\eta) G}{1-q \eta}\left[(1-\eta) q(D-G)+\underline{c}\left[(1-\eta) q+(1-\eta q) \frac{1-\tau}{\tau}\right]\right]}{[q(\tau q G-\underline{c})]^{2}}<0 .
$$

\section{Proof of Lemma 1}

To determine the value of the maximum payment that can be received by the experts, consider this constrained maximization problem:

$$
\max _{b \geq 0, w-u \geq 0} \tau b+(w-u)
$$

subject to (B12) and (B13) and let $\mu_{1}$ and $\mu_{2}$ be the Lagrangian multipliers associated with such constraints. Consider the solution where $b>0, w-u>0$ and (B13) binds. From the complementary slackness conditions, we get:

$$
\begin{aligned}
& \tau-\mu_{1} \delta(1-q \eta)=0 \\
& 1-\mu_{1}(1-\delta q \eta)=0 \\
& (1-\delta q \eta)(w-u)+\delta(1-q \eta) b-\delta q(1-\eta) G=0 .
\end{aligned}
$$

From the first two conditions we get that $\tau=\frac{\delta(1-q \eta)}{1-\delta q \eta}$, and then in the third equation:

$$
w-u+\tau b=\frac{\delta q(1-\eta) G}{1-\delta q \eta}
$$

For $\tau \neq \hat{\tau}(\delta)$, if (B13) binds, then either $b=0$ or $w-u=0 .{ }^{32}$ If $\tau>\hat{\tau}(\delta)$ paying only a bribe is desirable. To see this, substitute $w-u=\frac{\delta(1-q \eta) b}{(1-\delta q \eta)}-\frac{\delta q(1-\eta) G}{(1-\delta q \eta)}$ from (B13) into program (B15) and derive with respect to $b$. The derivative is strictly positive whenever $\tau>\hat{\tau}(\delta)$.

\footnotetext{
${ }^{32}$ Notice also that if both constraints bind, either $b$ or $w-u$ is strictly positive.
} 


\section{Proof of Corollary 1}

Suppose that (B13) does not hold when $w-u=\underline{c}$ and $b=0$ and set $w-u=\underline{c}-\epsilon$ and $b=\frac{\epsilon}{\tau}$, with $\epsilon>0$ so that (B10) binds in an attempt to satisfy (B13) :

$$
\begin{aligned}
w-u & =\underline{c}-\epsilon \leq \frac{\delta(1-q \eta)(\underline{c}-\epsilon)}{\tau(1-\delta q \eta)} \\
& \Leftrightarrow \tau(1-\delta q \eta) \leq \delta(1-q \eta),
\end{aligned}
$$

which is never the case because $\tau$ is, by assumption, higher.

\section{References}

Angelucci, C. and Russo, A. (2017). Petty corruption and citizen reports. CESifo Working Paper Series 5528.

Barbosa, K. and Straub, S. (2017). The value of revolving doors in public procurement. TSE Working Paper 17-873.

Barron, D. and Guo, Y. (2021). The use and misuse of coordinated punishments. The Quarterly Journal of Economics, 136(1):471-504.

Bertrand, M., Bombardini, M., and Trebbi, F. (2014). Is it whom you know or what you know? an empirical assessment of the lobbying process. American Economic Review, 104(12):3885-3920.

Blanes i Vidal, J., Draca, M., and Fons-Rosen, C. (2012). Revolving door lobbyists. The American Economic Review, 102(7):3731.

Bond, P. and Glode, V. (2014). The labor market for bankers and regulators. The Review of Financial Studies, 27(9):2539-2579.

Chassang, S. and Padró I Miquel, G. (2019). Crime, intimidation, and whistleblowing: A theory of inference from unverifiable reports. The Review of Economic Studies, 86(6):2530-2553.

Che, Y.-K. (1995). Revolving doors and the optimal tolerance for agency collusion. The RAND Journal of Economics, 26(3):378-397. 
Cornaggia, J., Cornaggia, K. J., and Xia, H. (2016). Revolving doors on wall street. Journal of Financial Economics, 120(2):400-419.

Darby, M. R. and Karni, E. (1973). Free competition and the optimal amount of fraud. Journal of Law and Economics, 16:67-88.

De Chiara, A. and Manna, E. (2019). Corruption and the regulation of innovation. UB Economics-Working Papers E19/390.

De Chiara, A. and Schwarz, M. A. (2020). A dynamic theory of regulatory capture. University of Innsbruck - Working Papers in Economics and Statistics 2020-12.

DeHaan, E., Kedia, S., Koh, K., and Rajgopal, S. (2015). The revolving door and the SEC's enforcement outcomes: Initial evidence from civil litigation. Journal of Accounting and Economics, 60(2-3):65-96.

Dranove, D. and Jin, G. Z. (2010). Quality disclosure and certification: Theory and practice. Journal of Economic Literature, 48(4):935-63.

Drugov, M. (2010). Competition in bureaucracy and corruption. Journal of Development Economics, 92(2):107-114.

Dulleck, U., Gong, J., and Li, J. (2015). Contracting for infrastructure projects as credence goods. Journal of Public Economic Theory, 17(3):328-345.

Dulleck, U. and Kerschbamer, R. (2006). On doctors, mechanics, and computer specialists: The economics of credence goods. Journal of Economic Literature, 44:5-42.

Estache, A. and Wren-Lewis, L. (2009). Toward a theory of regulation for developing countries: Following Jean-Jacques Laffont's lead. Journal of Economic Literature, $47(3): 729-70$.

Harstad, B. and Svensson, J. (2011). Bribes, lobbying, and development. American Political Science Review, pages 46-63.

Hiriart, Y. and Martimort, D. (2012). How much discretion for risk regulators? The RAND Journal of Economics, 43(2):283-314. 
Hiriart, Y., Martimort, D., and Pouyet, J. (2010). The public management of risk: Separating ex ante and ex post monitors. Journal of Public Economics, 94(11-12):10081019.

Inderst, R. (2015). Regulating commissions in markets with advice. International Journal of Industrial Organization, 43:137-141.

Inderst, R. and Ottaviani, M. (2012a). Competition through commissions and kickbacks. The American Economic Review, 102:780-809.

Inderst, R. and Ottaviani, M. (2012b). Financial advice. Journal of Economic Literature, 50:494-512.

Inderst, R. and Ottaviani, M. (2012c). How (not) to pay for advice: A framework for consumer financial protection. Journal of Financial Economics, 105:393-411.

Kempf, E. (2020). The job rating game: The effects of revolving doors and analyst incentives. Journal of Financial Economics, 135(1):41-67.

Khalil, F., Lawarrée, J., and Yun, S. (2010). Bribery versus extortion: allowing the lesser of two evils. The Rand journal of economics, 41(1):179-198.

Laffont, J.-J. and Martimort, D. (1999). Separation of regulators against collusive behavior. The Rand Journal of Economics, pages 232-262.

Laffont, J.-J. and Tirole, J. (1991). The politics of government decision-making: A theory of regulatory capture. The Quarterly Journal of Economics, 106(4):1089-1127.

Laffont, J.-J. and Tirole, J. (1993). A theory of incentives in procurement and regulation. MIT press.

Martimort, D. (1999). The life cycle of regulatory agencies: Dynamic capture and transaction costs. The Review of Economic Studies, 66(4):929-947.

Mulcahy, S. (2015). Lobbying in Europe. Transparency International.

Ortner, J. and Chassang, S. (2018). Making corruption harder: asymmetric information, collusion, and crime. Journal of Political Economy, 126(5):2108-2133. 
Piller, C. (2018). Hidden conflicts? Science, 361:16-20.

Salant, D. J. (1995). Behind the revolving door: A new view of public utility regulation. The RAND Journal of Economics, 26(3):362-377.

Stigler, G. J. (1971). The theory of economic regulation. The Bell Journal of Economics and Management Science, 2(1):3-21.

Tabakovic, H. and Wollmann, T. G. (2018). From revolving doors to regulatory capture? Evidence from patent examiners. NBER Working Paper No. 24638.

Tenekedjieva, A.-M. (2019). The revolving door and insurance solvency regulations. Working Paper.

Tirole, J. (1986). Hierarchies and bureaucracies: On the role of collusion in organizations. Journal of Law, Economics, $\mathscr{B}$ Organization, 2(2):181-214.

Tirole, J. (1992). Collusion and the theory of organizations. In Advances in Economic Theory, Vol. 2. Cambridge University Press.

Troya-Martinez, M. and Wren-Lewis, L. (2018). Managing relational contracts. CEPR Discussion Paper 12645. 